\title{
THE TUCANA/HOROLOGIUM, COLUMBA, AB DORADUS, AND ARGUS ASSOCIATIONS: NEW MEMBERS AND DUSTY DEBRIS DISKS
}

\author{
B. ZUCKERMAN ${ }^{1}$, JosePh H. RHEE ${ }^{1}$, INSEOK SONG ${ }^{2}$, AND M. S. BESSELL ${ }^{3}$ \\ ${ }^{1}$ Department of Physics and Astronomy, University of California, Los Angeles, CA 90095, USA; ben@ astro.ucla.edu, rhee@ astro.ucla.edu \\ ${ }^{2}$ Department of Physics and Astronomy, University of Georgia, Athens, GA 30602-2451, USA; song@ physast.uga.edu \\ ${ }^{3}$ Research School of Astronomy and Astrophysics, Australian National University, MSO, Cotter Road, Weston, ACT 2611, Australia; bessell@mso.anu.edu.au \\ Received 2011 January 31; accepted 2011 February 19; published 2011 April 15
}

\begin{abstract}
We propose 35 star systems within $\sim 70 \mathrm{pc}$ of Earth as newly identified members of nearby young stellar kinematic groups; these identifications include the first A- and late-B-type members of the AB Doradus moving group and field Argus Association. All but one of the 35 systems contain a bright solar- or earlier-type star that should make an excellent target for the next generation of adaptive optics (AO) imaging systems on large telescopes. AO imaging has revealed four massive planets in orbit around the $\lambda$ Boo star HR 8799. Initially, the planets were of uncertain mass due in large part to the uncertain age of the star. We find that HR 8799 is a likely member of the $\sim 30 \mathrm{Myr}$ old Columba Association, implying planet masses $\sim 6$ times that of Jupiter. We consider Spitzer Space Telescope MIPS photometry of stars in the $\sim 30$ Myr old Tucana/Horologium and Columba Associations, the $\sim 40$ Myr old field Argus Association, and the $\sim 70$ Myr old AB Doradus moving group. The percentage of stars in these young stellar groups that display excess emission above the stellar photosphere at 24 and $70 \mu \mathrm{m}$ wavelengths-indicative of the presence of a dusty debris disk-is compared with corresponding percentages for members of 11 open clusters and stellar associations with ages between 8 and $750 \mathrm{Myr}$, thus elucidating the decay of debris disks with time.
\end{abstract}

Key words: circumstellar matter - stars: evolution - stars: kinematics and dynamics - stars: pre-main sequence

Online-only material: color figures

\section{INTRODUCTION}

Young stars within $100 \mathrm{pc}$ of Earth are excellent laboratories for the study of stellar and planetary system evolution during ages from about ten to hundreds of millions of years. Except for the Sun, it is difficult to deduce even a moderately accurate age of an individual star. Astronomers have circumvented this problem by studying stars in rich open and globular clusters. Except for the Hyades, no such clusters exist within $100 \mathrm{pc}$ of Earth. Fortunately, beginning in the late 1990s, the existence of various substantial co-moving, co-eval, associations of young nearby stars has become evident (see reviews by Zuckerman \& Song 2004 and Torres et al. 2008).

Investigation of such stars will enhance knowledge of early stellar evolution. However, as a consequence of their proximity to Earth, in the long term the most valuable contribution of these youthful stars to astronomy will likely lie in the realm of the origin and early evolution of planetary systems - proximity buys one enhanced brightness and, for a given linear scale, an enhanced angular scale. Table 1 lists papers in which new members of the four nearest co-moving groups of young stars have been proposed previously; according to Torres et al. (2008), the mean distance from Earth is less than $50 \mathrm{pc}$ for the most secure members of each of these four groups. As new telescopes and cameras are being developed for imaging of young planets and the dusty disks out of which they form, it is important to complete the inventory of young stars near Earth.

A principal motivation of the present paper is the identification of new members of the AB Doradus and Tucana/ Horologium Associations. While considering the latter association, we recognized new members of the Columba Association (Torres et al. 2008) including the now famous HR 8799 (=HIP 114189) which is orbited by at least four giant planets (Marois et al. 2010). The age and some other properties of HR 8799 are considered in Section 5.3.
While considering the AB Dor Association we have identified its first known A- and late-B-type members. In addition, we have identified field members of the Argus Association that are of earlier spectral type and generally nearer to Earth than members previously proposed by Torres et al. (2008).

The Infrared Astronomical Satellite (IRAS) discovered the phenomenon of dusty debris disks in orbit around mainsequence stars (Aumann et al. 1984). By now $\sim 150$ mainsequence stars within $120 \mathrm{pc}$ of Earth appear to have excess infrared emission detectable with IRAS. Almost all of these excesses are measured most convincingly at $60 \mu \mathrm{m}$ wavelength (Moor et al. 2006; Rhee et al. 2007b, and references therein), although occasionally instead at $25 \mu \mathrm{m}$ (Rhee et al. 2007a; Melis et al. 2010, and references therein). While the Infrared Space Observatory (ISO) added a few new debris disk stars (e.g., Habing et al. 2001; Silverstone 2000), Spitzer Space Telescope surveys provided the next major advance; open clusters, nearby stellar associations, and nearby field stars have all been studied. Spitzer surveys included a variety of goals-for example, an understanding of the evolution of the quantity and temperature of the dusty debris with time, the association of debris disks with stars of different spectral classes, and association or lack thereof of debris with known planetary and stellar secondaries. IRAS was an all-sky survey whereas ISO and Spitzer pointed at only a small fraction of the sky. They were followed by the AKARI all-sky survey that, typically, was no more sensitive than IRAS. Most recently, the Wide Field Infrared Survey Explorer (WISE) has performed a mid-infrared all-sky survey far more sensitive than those of IRAS or AKARI.

A focus of the present paper is the evolution of debris disks as a function of their age. We present published and unpublished Spitzer data for Tucana/Horologium, Columba, $\mathrm{AB}$ Doradus, and Argus Association members, identifying some previously unrecognized dusty systems. Then we compare the overall debris disk status of these associations with previously 
Table 1

Papers Proposing Memberships in the Nearest Known Young Stellar Associations

\begin{tabular}{|c|c|c|c|c|}
\hline Paper & Tuc/Hor & AB Dor & TW Hya & $\beta$ Pic \\
\hline Kastner et al. (1997) & & & $\mathrm{X}$ & \\
\hline Webb et al. (1999) & & & $\mathrm{X}$ & \\
\hline Zuckerman \& Webb (2000) & $X$ & & & \\
\hline Torres et al. (2000) & $X$ & & & \\
\hline Zuckerman et al. (2001a) & & & & $\mathrm{X}$ \\
\hline Zuckerman et al. (2001b) & $\mathrm{X}$ & & & \\
\hline Zuckerman et al. (2001c) & & & $\mathrm{X}$ & \\
\hline Gizis (2002) & & & $\mathrm{X}$ & \\
\hline Song et al. (2003) & $X$ & & $\mathrm{X}$ & $\mathrm{X}$ \\
\hline Zuckerman et al. (2004) & & $\mathrm{X}$ & & \\
\hline Zuckerman \& Song (2004) & $\mathrm{X}$ & $\mathrm{X}$ & $\mathrm{X}$ & $\mathrm{X}$ \\
\hline Scholz et al. (2005) & & & $\mathrm{X}$ & \\
\hline Mamajek (2005) & & & $\mathrm{X}$ & \\
\hline Torres et al. (2006) & & & & $\mathrm{X}$ \\
\hline Lopez-Santiago et al. (2006) & & $\mathrm{X}$ & & \\
\hline Looper et al. (2007) & & & $\mathrm{X}$ & \\
\hline Torres et al. (2008) & $\mathrm{X}$ & $\mathrm{X}$ & $\mathrm{X}$ & $\mathrm{X}$ \\
\hline Fernandez et al. (2008) & $\mathrm{X}$ & $\mathrm{X}$ & $\mathrm{X}$ & $\mathrm{X}$ \\
\hline da Silva et al. (2009) & $\mathrm{X}$ & $\mathrm{X}$ & $\mathrm{X}$ & $\mathrm{X}$ \\
\hline Schlieder et al. (2010) & & $\mathrm{X}$ & & $\mathrm{X}$ \\
\hline Looper et al. (2010a, 2010b) & & & $X$ & \\
\hline Rodriguez et al. (2011) & & & $\mathrm{X}$ & \\
\hline Kiss et al. (2011) & $\mathrm{X}$ & & & $\mathrm{X}$ \\
\hline Shkolnik et al. (2011) & & & $X$ & \\
\hline This paper & $\mathrm{X}$ & $\mathrm{X}$ & & \\
\hline
\end{tabular}

Notes. In addition to the above major young stellar associations nearest to Earth, Zuckerman et al. (2006) proposed the somewhat older and sparser, but comparably nearby, "Carina-Near" moving group. The Carina Association proposed by Torres et al. (2008) has essentially nothing in common with the Carina-Near group; the latter is much nearer to Earth and much older than the former and has a much more negative $U$ component of space motion. While the Carina, Columba, and Argus Associations possess some members close to Earth (see, e.g., Tables 3 and 4), as defined by Torres et al. (2008), stars in these three associations are, on average, substantially more distant from Earth than are stars in the four Table 1 Associations (see Table 2 in Torres et al.).

published Spitzer results for other stellar associations near Earth.

\section{SAMPLE SELECTION}

Memberships of the four most prominent young stellar associations closest to Earth- $\beta$ Pic, TW Hya, AB Dor, and
Tuc/Hor-are considered in papers listed in Table 1. In the present paper, we address the latter two; specifically, we search for new members and for dusty debris disks. Although stars of the Columba Association are generally more distant from Earth than those of the four Table 1 Associations (Torres et al. 2008), because of various similarities between Tuc/Hor and Columba stars we searched for Columba stars within $65 \mathrm{pc}$ of Earth. The Argus Association, especially IC 2391, as proposed by Torres et al. (2008, their Tables 11 and 12) is on average much more distant from Earth than are the four Table 1 associations, but a few of their proposed Argus field members are within $70 \mathrm{pc}$ of Earth. This inspired us to search for additional nearby Argus field stars. Proposed new members of AB Dor, Tuc/Hor \& Columba, and Argus, all within $\sim 70$ pc of Earth, may be found in Tables 2-4.

Potential new members of these associations are identified by a match of ages and Galactic space motions $U V W$. These characteristics can be measured or estimated via optical spectroscopy and astrometry and also optical and X-ray photometry. Optical data for late-F through early-M-type stars come principally from our spectroscopic survey described in Section 3. For these spectral types, we generally observed only stars that appear in the ROSAT All Sky Survey (RASS; Voges et al. 1999, 2000). For earlier spectral types, an important source of data is the catalog of Gontcharov (2006) which includes accurate radial velocities for stars from late-B through early-F type.

For dusty debris disk studies with the Spitzer Space Telescope, MIPS photometry was obtained via our GO program no. 3600 "Disk Census of Nearby Stellar Groups," but also via the Spitzer archives. When poring through the Spitzer literature, we noticed that refereed papers had been devoted to most young and/or nearby stellar clusters and associations except that AB Dor and Tuc/Hor were conspicuous by their absence; we are now filling this void. Because some likely members of AB Dor and Tuc/Hor are identified in papers that post date the cold Spitzer mission (including some papers listed in Table 1 and also the present paper) infrared photometry for these two stellar associations is incomplete. Similar remarks pertain to Columba and Argus Association members. Perhaps WISE, Herschel, and SOFIA will help to complete the disk census for stars in young, nearby associations.

\section{OBSERVATIONS AND ANALYSIS}

For southern hemisphere stars, spectra were obtained with the $2.3 \mathrm{~m}$ telescope at the Siding Spring Observatory (SSO) of the Australian National University. Double-beam grating

Table 2

Proposed AB Doradus Moving Group Members

\begin{tabular}{lccccccrcc}
\hline \hline HIP & HD & $\begin{array}{c}\text { R.A. } \\
(\mathrm{h} / \mathrm{m})\end{array}$ & $\begin{array}{c}\text { Decl. } \\
(\mathrm{deg})\end{array}$ & $\begin{array}{c}\text { Spec. } \\
\text { Type }\end{array}$ & $\begin{array}{c}V \\
(\mathrm{mag})\end{array}$ & $\begin{array}{c}\text { Dist. } \\
(\mathrm{pc})\end{array}$ & $\begin{array}{c}\text { Rad. Vel. } \\
\left(\mathrm{km} \mathrm{s}^{-1}\right)\end{array}$ & $\begin{array}{c}(U, V, W) \\
\left(\mathrm{km} \mathrm{s}^{-1}\right)\end{array}$ & $\begin{array}{c}U V W \text { Error } \\
\left(\mathrm{km} \mathrm{s}^{-1}\right)\end{array}$ \\
\hline 13209 & 17573 & 0249 & +27 & B8 & 3.6 & 49 & $4.0 \pm 4.1$ & $-8.5,-25.7,-15.6$ & $3.2,1.9,2.0$ \\
15353 & 20888 & 0317 & -66 & A3 & 6.0 & 58 & $26.0 \pm 0.5$ & $-6.8,-27.5,-11.5$ & $0.4,0.5,0.4$ \\
93580 & 177178 & 1903 & +01 & A4 & 5.8 & 55 & $-23.1 \pm 2.3$ & $-11.3,-24.3,-12.8$ & $1.9,1.4,0.6$ \\
95347 & 181869 & 1923 & -40 & B8 & 4.0 & 52 & $-0.7 \pm 4.1$ & $-8.1,-26.0,-14.6$ & $3.8,1.4,1.8$ \\
109268 & 209952 & 2208 & -46 & B6 & 1.7 & 31 & $10.9 \pm 1.7$ & $-7.0,-25.6,-15.5$ & $1.1,0.7,1.4$ \\
115738 & 220825 & 2326 & +01 & A0 & 4.9 & 50 & $-4.4 \pm 0.6$ & $-7.0,-26.4,-13.3$ & $0.3,1.0,0.8$ \\
117452 & 223352 & 2348 & -28 & A0 & 4.6 & 44 & $8.7 \pm 2.0$ & $-7.0,-27.5,-13.3$ & $0.6,1.4,2.0$ \\
\hline
\end{tabular}

Notes. Input data (R.A., decl., distance, and proper motion) for the $U V W$ calculations in Tables 2-4 are from the Hipparcos catalog. UVW are defined with respect to the Sun, with $U$ positive toward the Galactic Center, $V$ positive in the direction of Galactic rotation, and $W$ positive toward the North Galactic pole. Characteristic mean $U V W$ for AB Dor group stars are given in Zuckerman \& Song (2004) as $-8,-27$, and $-14 \mathrm{~km} \mathrm{~s}^{-1}$, and in Torres et al. (2008) as $-6.8 \pm 1.3,-27.2 \pm 1.2$, and $-13.3 \pm 1.6 \mathrm{~km} \mathrm{~s}^{-1}$. HIP 93580 and 117452 are considered in Section 5.1. 
Table 3

Proposed Tucana/Horologium \& Columba Association Members

\begin{tabular}{|c|c|c|c|c|c|c|c|c|c|c|c|}
\hline HIP & HD & $\begin{array}{l}\text { R.A. } \\
(\mathrm{h} / \mathrm{m})\end{array}$ & $\begin{array}{l}\text { Decl. } \\
(\mathrm{deg})\end{array}$ & $\begin{array}{l}\text { Spec. } \\
\text { Type }\end{array}$ & $\begin{array}{c}V \\
(\mathrm{mag})\end{array}$ & $\begin{array}{l}\text { Dist. } \\
(\mathrm{pc}) \\
\end{array}$ & $\begin{array}{l}\text { Rad. Vel. } \\
\left(\mathrm{km} \mathrm{s}^{-1}\right)\end{array}$ & $\begin{array}{c}(U, V, W) \\
\left(\mathrm{km} \mathrm{s}^{-1}\right) \\
\end{array}$ & $\begin{array}{c}U V W \text { Error } \\
\left(\mathrm{km} \mathrm{s}^{-1}\right)\end{array}$ & $\begin{array}{c}\mathrm{Li} \text { EW } \\
(\mathrm{m} \AA)\end{array}$ & $f_{x}$ \\
\hline 1134 & 984 & 0014 & -07 & F5* & 7.3 & 46 & $-2.2 \pm 1.2$ & $-12.2,-23.2,-6.0$ & $0.7,1.3,1.2$ & 120 & -4.26 \\
\hline 12413 & 16754 & 0239 & -42 & $\mathrm{~A} 1 *$ & 4.7 & 40 & $18.0 \pm 4.2$ & $-10.8,-21.2,-8.3$ & $0.6,1.9,3.7$ & & \\
\hline 12925 & 17250 & 0246 & +05 & F8 & 7.9 & 63 & $4.3 \pm 1.1$ & $-10.8,-23.6,-0.6$ & $1.0,1.8,0.9$ & 145 & -4.33 \\
\hline 14551 & 19545 & 0307 & -27 & A5 & 6.2 & 58 & $13.8 \pm 0.8$ & $-10.8,-20.4,-3.2$ & $0.4,0.8,0.8$ & & \\
\hline 14913 & 20121 & 0312 & -44 & F6 & 5.9 & 44 & $13.5 \pm 2.1$ & $-10.8,-18.8,-2.3$ & $0.5,1.2,1.8$ & 65 & -4.06 \\
\hline 17248 & & 0341 & +55 & M0.5* & 11.2 & 37 & $-3.2 \pm 0.6$ & $-12.0,-23.5,-6.5$ & $1.2,1.7,0.6$ & & -3.36 \\
\hline 17764 & 24636 & 0348 & -74 & F3 & 7.1 & 55 & $15.5 \pm 1.3$ & $-8.6,-21.8,-2.5$ & $0.6,1.0,0.8$ & 60 & -5.4 \\
\hline 17782 & 23524 & 0348 & +52 & G8 & 8.8 & 51 & $-2.2 \pm 0.6$ & $-9.5,-20.1,-4.5$ & $1.8,2.9,1.0$ & 243 & -3.16 \\
\hline 17797 & $24071 \mathrm{~J}$ & 0348 & -37 & A1 & 4.7 & 49 & $15.6 \pm 0.4$ & $-10.2,-21.5,-1.0$ & $0.3,0.6,0.6$ & & \\
\hline 23179 & 31647 & 0459 & +37 & $\mathrm{~A} 1 *$ & 5.0 & 49 & $7.7 \pm 2.5$ & $-12.6,-22.2,-5.8$ & $2.5,1.2,0.3$ & & \\
\hline \multirow[t]{2}{*}{23362} & 32309 & 0501 & -20 & $\mathrm{~B} 9 *$ & 4.9 & 64 & $24.2 \pm 2.8$ & $-13.8,-22.6,-5.5$ & $1.8,1.6,1.5$ & & \\
\hline & 36869 & 0534 & -15 & G3* & 8.5 & (59) & $23.0 \pm 1.0$ & $-12,-21,-5$ & & 204 & -3.53 \\
\hline 26309 & 37286 & 0536 & -28 & $\mathrm{~A} 2 *$ & 6.3 & 57 & $22.4 \pm 1.2$ & $-11.3,-20.0,-4.9$ & $0.7,0.9,0.6$ & & \\
\hline 26453 & 37484 & 0537 & -28 & $\mathrm{~F} 3 *$ & 7.2 & 60 & $23.5 \pm 0.4$ & $-11.9,-20.9,-5.4$ & $0.3,0.4,0.4$ & 87 & -5.0 \\
\hline 26990 & 38397 & 0543 & -39 & G0* & 8.1 & 52 & $22.8 \pm 0.6$ & $-11.6,-20.3,-5.1$ & $0.3,0.5,0.4$ & 137 & -4.05 \\
\hline 28474 & 41071 & 0600 & -44 & G8* & 9.1 & 54 & $23.8 \pm 0.4$ & $-12.1,-21.1,-5.7$ & $0.3,0.4,0.5$ & 155 & -4.41 \\
\hline 32104 & 48097 & 0642 & +17 & $\mathrm{~A} 2 *$ & 5.2 & 43 & $15.0 \pm 4.2$ & $-10.4,-20.1,-4.9$ & $4.0,2.0,0.9$ & & \\
\hline 83494 & 154431 & 1703 & +34 & A5 & 6.1 & 54 & $-21.5 \pm 1.4$ & $-10.0,-24.3,-0.2$ & $0.7,1.1,1.0$ & & \\
\hline \multirow[t]{2}{*}{84642} & 155915 & 1718 & -60 & G8 & 9.5 & 55 & $1.3 \pm 0.7$ & $-12.6,-24.8,-1.1$ & $1.2,1.9,0.3$ & & \\
\hline & $* * B D+44$ & 2100 & +45 & $\mathrm{G} 2 *$ & 8.8 & (65) & $-23.2 \pm 1.5$ & $-11.0,-22.8,-8.2$ & & 196 & -3.48 \\
\hline 114189 & 218396 & 2307 & +21 & $\mathrm{~A} 5^{*}$ & 6.0 & 40 & $-12.6 \pm 1.4$ & $-12.3,-21.5,-7.2$ & $0.5,1.2,1.0$ & & \\
\hline 116805 & 222439 & 2340 & +44 & B9* & 4.1 & 52 & $-12.7 \pm 0.6$ & $-11.7,-20.3,-5.9$ & $0.6,0.8,0.4$ & & \\
\hline
\end{tabular}

Notes. $* * \mathrm{BD}+44=\mathrm{BD}+443670$. In the fifth column, a * after a spectral type indicates a suggested Columba Association member. The listed distances to HD 36869 and BD+44 3670 are derived photometrically. Tycho-2 proper motions are used in the calculation of $U V W$ for these two stars. The $U V W$ of HIP 14551 and 17782 appear to be a mixture of those of Columba and Tuc/Hor. Characteristic mean $U V W$ for Tuc/Hor stars are given in Zuckerman \& Song (2004) as $-11,-21$, and $0 \mathrm{~km} \mathrm{~s}^{-1}$, and in Torres et al. (2008) as $-9.9 \pm 1.5,-20.9 \pm 0.8$, and $-1.4 \pm 0.9 \mathrm{~km} \mathrm{~s}^{-1}$. Torres et al. give a mean $U V W$ for Columba stars of $-13.2 \pm 1.3,-21.8 \pm 0.8$, and $-5.9 \pm 1.2 \mathrm{~km} \mathrm{~s}^{-1}$. See Sections 4 and 5.2 for additional details regarding Table 3 stars. HIP $114189(=$ HR 8799 , see Section 5.3) is orbited by at least four giant planets.

Table 4

Proposed Argus Association Members

\begin{tabular}{lccccccccc}
\hline \hline HIP & HD & $\begin{array}{c}\text { R.A. } \\
(\mathrm{h} / \mathrm{m})\end{array}$ & $\begin{array}{c}\text { Decl. } \\
(\mathrm{deg})\end{array}$ & $\begin{array}{c}\text { Spec. } \\
\text { Type }\end{array}$ & $\begin{array}{c}V \\
(\mathrm{mag})\end{array}$ & $\begin{array}{c}\text { Dist. } \\
(\mathrm{pc})\end{array}$ & $\begin{array}{c}\text { Rad. Vel. } \\
\left(\mathrm{km} \mathrm{s}^{-1}\right)\end{array}$ & $\begin{array}{c}(U, V, W) \\
\left(\mathrm{km} \mathrm{s}^{-1}\right)\end{array}$ & $\begin{array}{c}U V W \text { Error } \\
\left(\mathrm{km} \mathrm{s}^{-1}\right)\end{array}$ \\
\hline 50191 & 88955 & 1014 & -42 & A2 & 3.8 & 31 & $7.4 \pm 2.7$ & $-22.0,-10.5,-4.9$ & $0.5,2.6,0.6$ \\
57632 & 102647 & 1149 & +14 & A3 & 2.1 & 11 & $-0.2 \pm 0.5$ & $-20.1,-16.2,-7.6$ & $0.2,0.2,0.5$ \\
68994 & 123058 & 1407 & -61 & F4 & 7.8 & 66 & $-5.2 \pm 1.0$ & $-21.2,-12.1,-2.5$ & $1.4,1.4,0.3$ \\
79797 & 145689 & 1617 & -67 & A4 & 6.0 & 55 & $-9.0 \pm 4.3$ & $-23.2,-12.0,-4.9$ & $3.3,2.7,1.0$ \\
98495 & 188228 & 2000 & -72 & A0 & 4.0 & 32 & $-6.7 \pm 0.7$ & $-21.8,-10.8,-4.5$ & $0.6,0.5,0.4$ \\
99770 & 192640 & 2014 & +36 & A2 & 5.0 & 41 & $-17.3 \pm 2.8$ & $-22.5,-11.7,-3.9$ & $0.8,2.7,0.1$ \\
\hline
\end{tabular}

Notes. Additional information regarding the listed stars can be found in Section 5.4. Torres et al. (2008) give a mean $U V W$ for Argus stars of $-22.0 \pm 0.3,-14.4 \pm 1.3$, and $-5.0 \pm 1.3 \mathrm{~km} \mathrm{~s}^{-1}$.

(DBS) and echelle spectrographs were employed at the two Nasmyth foci. The red channel of the DBS covers the spectral range $6500-7450 \AA$ at a measured resolution of $1.16 \AA$ $\left(0.55 \AA\right.$ pixel $\left.^{-1}\right)$. Eight orders of the echelle spectra cover portions of the wavelength range from 5800 to $7230 \AA$. We focus on orders that contain the $\mathrm{H} \alpha$ and $\mathrm{Li} \lambda 6708$ lines. In these orders, the measured resolution was $0.45 \AA\left(0.17 \AA\right.$ pixel $\left.^{-1}\right)$. Radial velocities were determined by cross-correlating target and radial velocity standard spectra over five or six orders of the echelle that were chosen to produce strong correlations and have few atmospheric features. All spectra were reduced following a standard procedure (bad pixel and cosmic ray removal, flat fielding, source extraction, telluric correction, etc.) using IRAF. Equivalent widths (EWs) of $\mathrm{H} \alpha$ and $\mathrm{Li} \lambda 6708$ were measured with the IRAF task splot.

For northern hemisphere stars, we used the Hamilton echelle spectrograph at the coude focus of the $3 \mathrm{~m}$ telescope at Lick
Observatory. Data reduction procedures were similar to those employed at Siding Spring.

As noted in Section 2, MIPS photometry was obtained via GO program no. 3600 and also via the Spitzer archives. For the MIPS $24 \mu \mathrm{m}$ band, we performed aperture photometry on post-BCD images provided by the Spitzer archive using an aperture radius of $13^{\prime \prime}$ and sky annuli of $20^{\prime \prime}$ and $32^{\prime \prime}$. An aperture correction of 1.17 was applied based on Table 4.13 of the MIPS Instrument Handbook version 2.0. In cases where the target is a binary object (e.g., HIP 116748), we used a larger aperture size to collect the combined Spitzer flux densities and compared them with combined optical and near-IR flux densities. For the MIPS $70 \mu \mathrm{m}$ band, we first created a mosaic image by combining BCD images with the Spitzer MOPEX tool and performed aperture photometry on the mosaic image. For a few stars, Spitzer Infrared Spectrograph (IRS) data exist and we used these where appropriate to confirm or deny the existence of excess 
Table 5

AB Doradus Stars Observed by Spitzer

\begin{tabular}{|c|c|c|c|c|c|c|c|c|c|c|c|}
\hline \multirow[t]{2}{*}{ HIP } & \multirow[t]{2}{*}{$\mathrm{HD}$} & \multirow[t]{2}{*}{ Name } & \multirow{2}{*}{$\begin{array}{l}\text { Spect. } \\
\text { Type }\end{array}$} & \multirow{2}{*}{$\begin{array}{l}\text { Dist. } \\
\text { (pc) }\end{array}$} & \multirow[t]{2}{*}{ ZS04 } & \multirow[t]{2}{*}{ T08 } & \multirow{2}{*}{$\begin{array}{c}\text { Spitzer } \\
\text { Program ID }\end{array}$} & \multirow{2}{*}{$\begin{array}{l}\text { AORKEY } \\
\text { Archive ID }\end{array}$} & \multirow{2}{*}{$\begin{array}{c}\text { Date of } \\
\text { Observation }\end{array}$} & \multicolumn{2}{|c|}{ Integ. Time (s) } \\
\hline & & & & & & & & & & $24 \mu \mathrm{m}$ & $70 \mu \mathrm{m}$ \\
\hline & 1405 & PW And & K2 & (28) & $\mathrm{Y}$ & $\mathrm{Y}$ & 3600 & 11254784 & 2004 Dec 25 & 48 & 252 \\
\hline 3589 & 4277 & & F8 & 49 & $\mathrm{Y}$ & $\mathrm{Y}$ & 3600 & 11255040 & 2005 Jan 28 & 48 & 252 \\
\hline 5191 & 6569 & & $\mathrm{~K} 1$ & 50 & $\mathrm{Y}$ & & 3600 & 11255296 & 2004 Dec 25 & 48 & 378 \\
\hline 6276 & & BD-12 243 & G9 & 35 & $\mathrm{Y}$ & $\mathrm{Y}$ & 148 & 5346304 & $2004 \mathrm{Jul} 11$ & 96 & 252 \\
\hline 10272 & 13482 & & K1 & 32 & $\mathrm{Y}$ & $\mathrm{Y}$ & 3600 & 11255808 & 2005 Jan 29 & 48 & 252 \\
\hline 13027 & 17332 & & G0 & 33 & $\mathrm{Y}$ & $\mathrm{Y}$ & 3600 & 11256832 & 2005 Jan 26 & 48 & 252 \\
\hline 14684 & 19668 & IS Eri & G0 & 40 & & $\mathrm{Y}$ & 148 & 5340928 & 2005 Jan 24 & 96 & 252 \\
\hline 14807 & & $\mathrm{BD}+21418 \mathrm{~B}$ & K6 & 50 & $\mathrm{Y}$ & $\mathrm{Y}$ & 72 & 4541952 & 2004 Feb 24 & 332 & 630 \\
\hline 14809 & & $\mathrm{BD}+21418$ & G5 & 50 & $\mathrm{Y}$ & $\mathrm{Y}$ & 72 & 4541952 & $2004 \mathrm{Feb} 24$ & 332 & 630 \\
\hline 15353 & 20888 & & $\mathrm{~A} 3$ & 58 & & & 10 & 3697408 & 2004 Nov 7 & 48 & 126 \\
\hline \multirow[t]{2}{*}{16563} & 21845 & V577 Per & G5 & 34 & $\mathrm{Y}$ & $\mathrm{Y}$ & 3600 & 11257600 & 2005 Feb 1 & 48 & 252 \\
\hline & $21845 B$ & & M0 & 34 & $\mathrm{Y}$ & $\mathrm{Y}$ & & & & & \\
\hline 17695 & & G80-21 & M3 & 16 & $\mathrm{Y}$ & $\mathrm{Y}$ & 3600 & 11258368 & 2004 Sep 23 & 48 & 378 \\
\hline 18859 & 25457 & HR 1249 & F5 & 19 & $\mathrm{Y}$ & $\mathrm{Y}$ & 148 & 5308672 & 2004 Sep 20 & 96 & 252 \\
\hline 19183 & 25953 & & F5 & 55 & $\mathrm{Y}$ & $\mathrm{Y}$ & 20707 & 15009792 & $2006 \mathrm{Feb} 21$ & 96 & 252 \\
\hline 25283 & 35650 & & K7 & 18 & $\mathrm{Y}$ & & 3600 & 11260416 & 2005 Mar 7 & 48 & 252 \\
\hline \multirow[t]{2}{*}{25647} & $36705 \mathrm{~A}$ & AB Dor & K0 & 15 & $\mathrm{Y}$ & $\mathrm{Y}$ & 80 & 4638720 & 2004 Feb 21 & 48 & 252 \\
\hline & 36705B & AB DorB & M4 & 15 & $\mathrm{Y}$ & $\mathrm{Y}$ & & & & & \\
\hline 26369 & & & K7 & 24 & $\mathrm{Y}$ & $\mathrm{Y}$ & 148 & 6599680 & 2004 Sep 21 & 96 & 252 \\
\hline \multirow[t]{2}{*}{26373} & 37572 & UY Pic & K0 & 24 & $\mathrm{Y}$ & $\mathrm{Y}$ & 148 & 6599680 & 2004 Sep 21 & 96 & 252 \\
\hline & & CD-35 2722 & M1 & (24) & & $\mathrm{Y}$ & 731 & 26807552 & 2008 Nov 25 & 664 & 1260 \\
\hline \multirow[t]{2}{*}{30314} & 45270 & & G1 & 23 & $\mathrm{Y}$ & $\mathrm{Y}$ & 148 & 6599424 & 2004 Jun 22 & 96 & 252 \\
\hline & & GSC 08894-0426 & M3 & (22) & $\mathrm{Y}$ & $\mathrm{Y}$ & 3600 & 11262464 & 2004 Dec 25 & 48 & 378 \\
\hline 31711 & 48189 & AK Pic & $\mathrm{G} 2$ & 22 & $\mathrm{Y}$ & $\mathrm{Y}$ & 80 & 4639232 & $2204 \mathrm{Feb} 21$ & 48 & 252 \\
\hline \multirow[t]{2}{*}{31878} & & CD-61 1439 & K7 & 22 & $\mathrm{Y}$ & $\mathrm{Y}$ & 3600 & 11262720 & 2004 Nov 8 & 48 & 252 \\
\hline & & $\mathrm{BD}+201790$ & K5 & (26) & & $\mathrm{Y}$ & 148 & 5348608 & 2004 Oct 14 & 96 & 1260 \\
\hline 36349 & & V372 Pup & M2 & 15 & $\mathrm{Y}$ & $\mathrm{Y}$ & 3600 & 11264000 & 2004 Nov 7 & 48 & 252 \\
\hline 51317 & & GJ 393 & M2 & 7 & & $\mathrm{Y}$ & 40454 & 22014720 & 2009 Jan 2 & 48 & 756 \\
\hline 63742 & 113449 & PX Vir & $\mathrm{K} 1$ & 22 & $\mathrm{Y}$ & $\mathrm{Y}$ & 80 & 4627968 & 2005 Jun 22 & 48 & 252 \\
\hline 76768 & 139751 & & K5 & 42 & $\mathrm{Y}$ & $\mathrm{Y}$ & 3600 & 11265536 & 2005 Aug 26 & 48 & 252 \\
\hline 81084 & & NLTT 43056 & M0 & 32 & $\mathrm{Y}$ & $\mathrm{Y}$ & 3600 & 11265792 & 2005 Mar 10 & 48 & 378 \\
\hline \multirow[t]{2}{*}{82688} & 152555 & & G0 & 48 & $\mathrm{Y}$ & $\mathrm{Y}$ & 148 & 5330944 & 2005 Mar 10 & 96 & 252 \\
\hline & 317617 & & $\mathrm{~K} 3$ & $(56)$ & & $\mathrm{Y}$ & 30594 & 20485632 & 2006 Oct 7 & 30 & 15 \\
\hline 86346 & 160934 & GJ 4020A & K7 & 28 & $\mathrm{Y}$ & $\mathrm{Y}$ & 72 & 4554240 & 2004 Jan 29 & 192 & 630 \\
\hline 93580 & 177178 & & A4 & 55 & & & 10 & 3724544 & 2004 Apr 11 & 48 & 126 \\
\hline 106231 & & LO Peg & K7 & 25 & $\mathrm{Y}$ & $\mathrm{Y}$ & 80 & 4641024 & 2004 Jun 22 & 48 & 630 \\
\hline 107948 & & GJ 4231 & M2 & 30 & & $\mathrm{Y}$ & 50356 & 26063616 & 2008 Jun 27 & 48 & 252 \\
\hline 109268 & 209952 & & B6 & 31 & & & 713 & 7345152 & 2003 Nov 3 & 96 & 114 \\
\hline 110526 & & GJ 856 & M3 & 16 & $\mathrm{Y}$ & $\mathrm{Y}$ & 3600 & 11266560 & 2004 Dec 2 & 48 & 378 \\
\hline 113579 & 217343 & & G4 & 32 & $\mathrm{Y}$ & $\mathrm{Y}$ & 148 & 5269760 & 2004 Jun 21 & 96 & 252 \\
\hline 113597 & 217379 & & K8 & 31 & $\mathrm{Y}$ & $\mathrm{Y}$ & 123 & 5022976 & 2004 Nov 2 & 144 & 630 \\
\hline 114066 & & GJ 9809 & M1 & 25 & $\mathrm{Y}$ & $\mathrm{Y}$ & 3600 & 11266816 & 2004 Oct 15 & 48 & 378 \\
\hline 114530 & 218860 & & G5 & 51 & $\mathrm{Y}$ & $\mathrm{Y}$ & 3600 & 11267072 & 2004 Oct 19 & 48 & 252 \\
\hline 115738 & 220825 & & $\mathrm{~A} 0$ & 50 & & & 171 & 3731456 & $2004 \mathrm{Jul} 11$ & 48 & 126 \\
\hline 117452 & 220825 & & $\mathrm{~A} 0$ & 44 & & & 173 & 3731968 & 2004 Dec 2 & 48 & 126 \\
\hline 118008 & 224228 & GJ 4377 & $\mathrm{~K} 2$ & 22 & $\mathrm{Y}$ & $\mathrm{Y}$ & 3600 & 11267584 & 2004 Nov 4 & 48 & 252 \\
\hline
\end{tabular}

Note. Distances in parenthesis are estimated photometrically. ZS04 and T08 refer to AB Dor members listed in Zuckerman \& Song (2004) and Torres et al. (2008), respectively.

IR emission. Spitzer results are summarized in Tables 5-11. Figures 1-7 display the spectral energy distributions (SEDs) of all AB Dor, Tuc/Hor, nearby Columba, and nearby Argus field stars that we deem to definitely or probably have excess infrared emission.

A fully automated SED-fitting technique employing a theoretical atmospheric model (Hauschildt et al. 1999) was used to predict stellar photospheric fluxes. A detailed description of our photospheric fitting procedure is given in Section 2 of Rhee et al. (2007b), so we do not repeat it here. To check whether our atmospheric model well reproduces actual stellar photospheres, in Figure 8 we compare the model predictions with MIPS mea- surements at $24 \mu \mathrm{m}$ of a sample of nearby F-, G-, and K-type stars from a paper by Trilling et al. (2008). Stars plotted in Figure 8 do not include any stars deemed by Trilling et al. to have either 24 or $70 \mu \mathrm{m}$ excess emission (their Tables 5 and 6). The plotted stars are all fifth magnitude or fainter at the $K$ band as measured in the Two Micron All Sky Survey (2MASS) survey. We avoid brighter stars that may be too bright to be measured accurately by 2MASS in the $J$ band. As may be seen in Figure 8 for $\mathrm{K}-, \mathrm{G}-$, and F-type stars the MIPS measured $24 \mu \mathrm{m}$ fluxes are on average about 3\% larger than the model predicted fluxes. While some of this offset may be due to small and previously unrecognized excess IR emission from some stars in the Trilling et al. 
Table 6

Tucana/Horologium \& Columba Stars Observed by Spitzer

\begin{tabular}{|c|c|c|c|c|c|c|c|c|c|c|c|}
\hline \multirow[t]{2}{*}{ HIP } & \multirow[t]{2}{*}{ HD } & \multirow[t]{2}{*}{ Name } & \multirow{2}{*}{$\begin{array}{l}\text { Spect. } \\
\text { Type }\end{array}$} & \multirow{2}{*}{$\begin{array}{l}\text { Dist. } \\
(\mathrm{pc})\end{array}$} & \multirow[t]{2}{*}{ ZS04 } & \multirow[t]{2}{*}{ T08 } & \multirow{2}{*}{$\begin{array}{c}\text { Spitzer } \\
\text { Program ID } \\
\end{array}$} & \multirow{2}{*}{$\begin{array}{l}\text { AORKEY } \\
\text { Archive ID }\end{array}$} & \multirow{2}{*}{$\begin{array}{c}\text { Date of } \\
\text { Observation }\end{array}$} & \multicolumn{2}{|c|}{ Integ. Time (s) } \\
\hline & & & & & & & & & & $24 \mu \mathrm{m}$ & $70 \mu \mathrm{m}$ \\
\hline 490 & 105 & & G0 & 40 & $\mathrm{Y}$ & $\mathrm{Y}$ & 148 & 5295872 & 2003 Dec 10 & 96 & 252 \\
\hline 1113 & 987 & & G7 & 44 & $\mathrm{Y}$ & $\mathrm{Y}$ & 102 & 9022976 & 2004 Nov 5 & 48 & 126 \\
\hline 1481 & 1466 & & F8 & 41 & $\mathrm{Y}$ & $\mathrm{Y}$ & 72 & 4539648 & 2004 Dec 2 & 96 & 630 \\
\hline 1910 & & & M1 & 45 & $\mathrm{Y}$ & $\mathrm{Y}$ & 72 & 4539904 & 2004 Jun 22 & 332 & 630 \\
\hline 1993 & & CT Tuc & M0 & 40 & Y & Y & 72 & 4540160 & 2004 Jun 22 & 332 & 630 \\
\hline 2484 & 2884 & beta1 Tuc & B9 & 43 & $\mathrm{Y}$ & Y & 72 & 4540416 & 2004 May 11 & 48 & 252 \\
\hline 2487 & 2885 & beta2 Tuc & A2 & 50 & $\mathrm{Y}$ & $\mathrm{Y}$ & 72 & 4540672 & 2004 May 5 & 48 & 252 \\
\hline 2578 & 3003 & beta3 Tuc & A0 & 46 & $\mathrm{Y}$ & $\mathrm{Y}$ & 72 & 4540928 & 2004 Jun 22 & 48 & 504 \\
\hline 2729 & 3221 & & K5 & 46 & $\mathrm{Y}$ & Y & 72 & 4541184 & 2004 May 11 & 192 & 630 \\
\hline \multirow[t]{2}{*}{3556} & & & M3 & 38 & $\mathrm{Y}$ & & 102 & 9022720 & 2004 Nov 3 & 48 & 252 \\
\hline & & CPD-64 120 & $\mathrm{~K} 1$ & (73) & $\mathrm{Y}$ & & 102 & 9022464 & 2004 May 11 & 48 & $\mathrm{~N} / \mathrm{A}$ \\
\hline 6485 & 8558 & & G6 & 49 & $\mathrm{Y}$ & $\mathrm{Y}$ & 148 & 4813568 & 2005 Jun 29 & 48 & 126 \\
\hline 6856 & 9054 & CC Phe & $\mathrm{K} 1$ & 37 & Y & Y & 149 & 4813824 & 2004 Nov 3 & 48 & 126 \\
\hline 9141 & 12039 & DK Cet & G4 & 42 & $\mathrm{Y}$ & $\mathrm{Y}$ & 148 & 5310976 & 2004 Jul 11 & 96 & 252 \\
\hline 9685 & 12894 & & F3 & 47 & $\mathrm{Y}$ & & 102 & 9022208 & 2004 Nov 7 & 48 & 126 \\
\hline 9892 & 13183 & & G6 & 50 & $\mathrm{Y}$ & $\mathrm{Y}$ & 152 & 4814592 & 2004 Nov 7 & 48 & 126 \\
\hline 9902 & 13246 & & F7 & 45 & $\mathrm{Y}$ & Y & 153 & 4814848 & 2004 Nov 7 & 48 & 126 \\
\hline & & CD-60 416 & K4 & $(48)$ & $\mathrm{Y}$ & $\mathrm{Y}$ & 153 & 4814848 & 2004 Nov 7 & 48 & 126 \\
\hline 10602 & 14228 & phi Eri & B8 & 47 & $\mathrm{Y}$ & Y & 102 & 9021952 & 2004 Nov 8 & 48 & 252 \\
\hline 12394 & 16978 & eps Hyi & B9 & 47 & $\mathrm{Y}$ & $\mathrm{Y}$ & 102 & 9021184 & 2005 Jun 19 & 48 & 252 \\
\hline 12413 & 16754 & & A1 & 40 & & & 10 & 3694848 & 2005 Jul 30 & 48 & 126 \\
\hline & & CD-53 544 & K6 & (30) & $\mathrm{Y}$ & $\mathrm{Y}$ & 154 & 4815104 & 2004 Nov 7 & 48 & 126 \\
\hline & & AF Hor & M2 & (27) & $\mathrm{Y}$ & Y & 154 & 4815104 & 2004 Nov 7 & 48 & 12 \\
\hline & & CD-58 553 & K5 & (50) & $\mathrm{Y} ?$ & Y & 155 & 4815360 & 2004 Nov 7 & 48 & 126 \\
\hline 14551 & 19545 & & A5 & 58 & & & 10 & 3696896 & 2006 Jan 13 & 48 & 126 \\
\hline 15247 & 20385 & & F5 & 50 & $\mathrm{Y}$ & & 3600 & 11257088 & 2005 Jan 31 & 48 & 252 \\
\hline & & CD-46 1064 & $\mathrm{~K} 3$ & 50 & & $\mathrm{Y}$ & 3600 & 11257344 & 2004 Oct 14 & 48 & 252 \\
\hline 16449 & 21997 & HR 1082 & A3 & 73 & & $\mathrm{Y}^{*}$ & 10 & 3698432 & 2004 Sep 24 & 48 & 126 \\
\hline 16853 & 22705 & & $\mathrm{G} 2$ & 42 & $\mathrm{Y}$ & $\mathrm{Y}$ & 3600 & 11258112 & 2004 Oct 14 & 48 & 252 \\
\hline 17764 & 24636 & & $\mathrm{~F} 3$ & 55 & & & 40566 & 23051520 & 2008 May 16 & 96 & 504 \\
\hline & & BD-15 705 & K3 & (63) & $\mathrm{Y}$ & $\mathrm{Y}^{*}$ & 3600 & 11258880 & 2005 Feb 1 & 48 & 278 \\
\hline 21632 & 29615 & & G3 & 55 & $\mathrm{Y}$ & $\mathrm{Y}$ & 3600 & 11259392 & 2005 Mar 4 & 48 & 252 \\
\hline 21965 & 30051 & & $\mathrm{~F} 2$ & 58 & $\mathrm{Y}$ & $\mathrm{Y}$ & 3600 & 11259648 & 2005 Jan 28 & 48 & 252 \\
\hline 22295 & 32195 & & F7 & 60 & $\mathrm{Y}$ & & 20707 & 15009536 & 2005 Aug 31 & 96 & 252 \\
\hline 24947 & 35114 & AS Col & F6 & 46 & Y & $\mathrm{Y}^{*}$ & 3600 & 11260160 & 2004 Dec 5 & 48 & 252 \\
\hline 23179 & 31647 & & A1 & 49 & & & 10 & 3699712 & 2004 Feb 25 & 48 & 126 \\
\hline & 36869 & & G3 & (59) & & & 3600 & 11260672 & 2005 Mar 4 & 48 & 126 \\
\hline 26309 & 37286 & & $\mathrm{~A} 2$ & 57 & & & 10 & 3700992 & 2004 Feb 21 & 48 & 126 \\
\hline & & AT Col & K1 & (57) & $\mathrm{Y}$ & $\mathrm{Y}^{*}$ & 3600 & 11260928 & 2005 Feb 28 & 48 & 278 \\
\hline 26453 & 37484 & & F3 & 60 & & & 148 & 5307136 & 2004 Nov 5 & 96 & 252 \\
\hline & & CD-34 2406 & K4 & $(60)$ & $\mathrm{Y}$ & & 3600 & 11261440 & 2005 Feb 28 & 48 & 278 \\
\hline 26966 & 38206 & HR 1975 & A0 & 69 & & $\mathrm{Y}^{*}$ & 40 & 3983872 & 2004 Feb 21 & 48 & 252 \\
\hline 28036 & 40216 & & F7 & 54 & $\mathrm{Y}$ & $\mathrm{Y}^{*}$ & 3600 & 11261952 & 2005 Mar 7 & 48 & 252 \\
\hline 30030 & 43989 & V1358 Ori & G0 & 50 & $\mathrm{Y}$ & & 148 & 6598912 & 2004 Oct 13 & 96 & 252 \\
\hline 30034 & 44627 & AB Pic & $\mathrm{K} 2$ & 45 & $\mathrm{Y}$ & & 3600 & 11262208 & 2004 Dec 25 & 48 & 252 \\
\hline 32104 & 48097 & & A2 & 43 & & & 10 & 3702784 & 2004 Mar 15 & 48 & 126 \\
\hline 32235 & 49855 & & G6 & 56 & $\mathrm{Y}$ & $\mathrm{Y}^{*}$ & 3600 & 11263488 & 2004 Nov 8 & 48 & 252 \\
\hline 32435 & 53842 & & F5 & 57 & $\mathrm{Y}$ & & 20707 & 15003648 & 2005 Aug 31 & 96 & 76 \\
\hline 33737 & 55279 & & $\mathrm{~K} 2$ & 64 & $\mathrm{Y}$ & $\mathrm{Y}^{*}$ & 3600 & 11263744 & 2005 Mar 8 & 48 & 278 \\
\hline 83494 & 154431 & & A5 & 54 & & & 10 & 3721472 & 2004 Feb 22 & 48 & 126 \\
\hline 84642 & 155915 & & G8 & 55 & & & 3600 & 11266048 & 2005 Apr 6 & 48 & 278 \\
\hline 100751 & 193924 & Peacock & B2 & 56 & $\mathrm{Y}$ & & 72 & 4557312 & 2004 Apr 9 & 48 & 126 \\
\hline 104308 & 200798 & & A5 & 66 & $\mathrm{Y}$ & & 72 & 4558592 & 2004 Sep 23 & 96 & 630 \\
\hline 105388 & 202917 & & G7 & 46 & $\mathrm{Y}$ & $\mathrm{Y}$ & 72 & 4558848 & 2004 Sep 24 & 192 & 630 \\
\hline 105404 & 202947 & BS Ind & K0 & 46 & $\mathrm{Y}$ & & 72 & 4559104 & 2004 Sep 24 & 192 & 630 \\
\hline 107345 & & & M0 & 42 & $\mathrm{Y}$ & Y & 72 & 4559360 & 2004 Sep 23 & 498 & 630 \\
\hline 107947 & 207575 & & F6 & 45 & Y & Y & 72 & 4560128 & 2004 Sep 23 & 498 & 630 \\
\hline 108195 & 207964 & HR 8352 & $\mathrm{~F} 1$ & 46 & $\mathrm{Y}$ & $\mathrm{Y}$ & 72 & 4560384 & 2004 Sep 23 & 498 & 630 \\
\hline 108422 & 208233 & & G9 & 55 & $\mathrm{Y}$ & & 3600 & 11266304 & 2004 Nov 6 & 48 & 252 \\
\hline 116748 & 222259 & DS Tuc & G6 & 46 & $\mathrm{Y}$ & & 72 & 4561920 & 2204 May 5 & 192 & 630 \\
\hline 118121 & 224392 & eta Tuc & A1 & 49 & $\mathrm{Y}$ & & 72 & 4562176 & 2004 Jun 22 & 48 & 630 \\
\hline
\end{tabular}

Notes. Distances in parenthesis are estimated photometrically. Y* in the T08 column indicates that Torres et al. (2008) place the star in what they designate as either the Columba or Carina Association. Because these associations have the same age and nearly the same $U V W$ space motions as Tucana/Horologium, for the purposes of Table 11 of the present paper we consider as a single age group the stars in all of these associations. Spitzer observations of HIP 114189 (= HR 8799, Table 3) have been analyzed in detail by Su et al. (2009) and we have therefore not included this star in Table 6. 

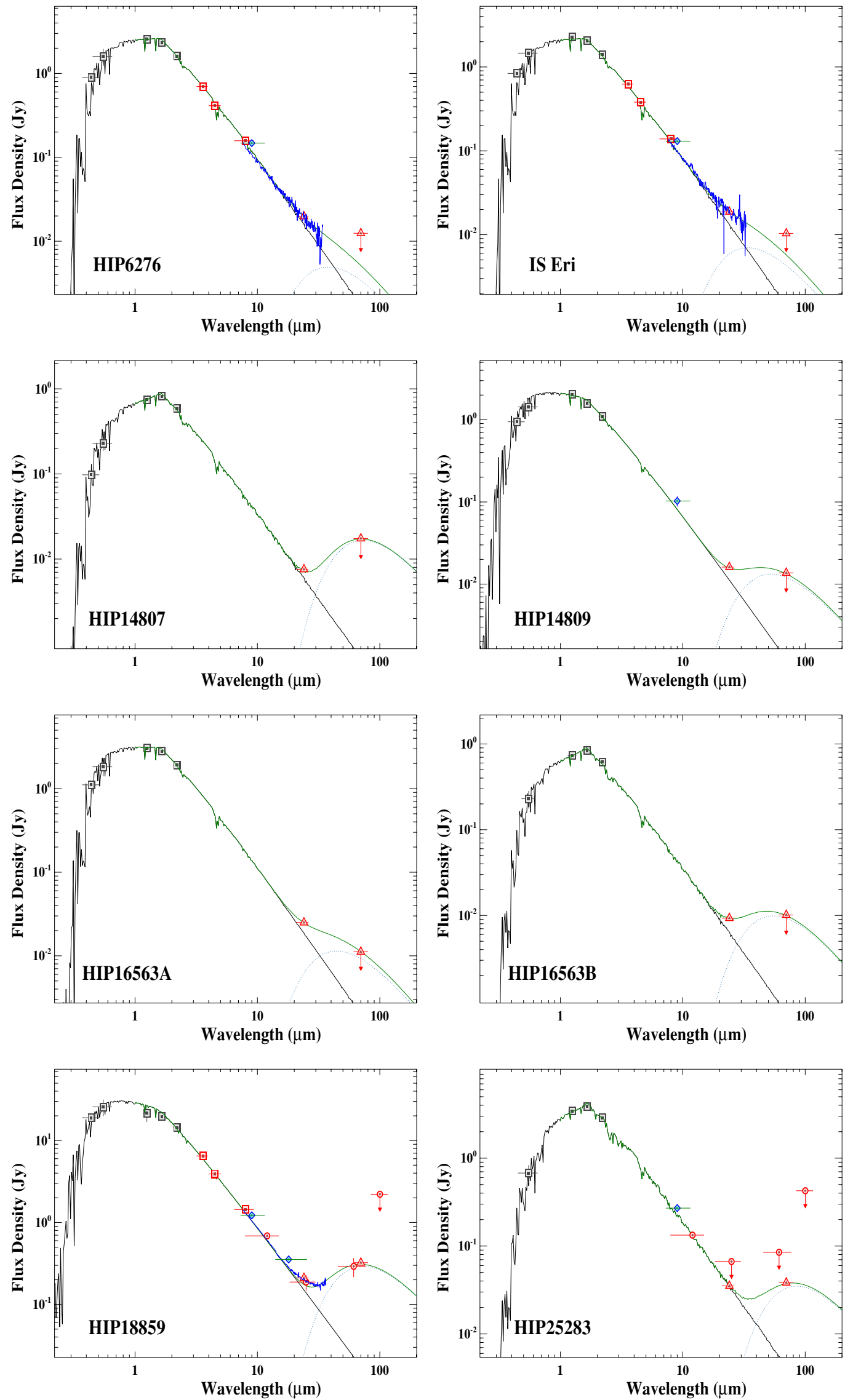

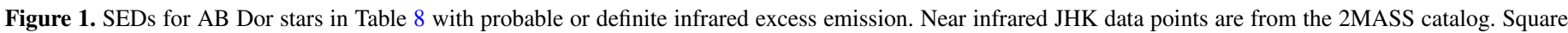

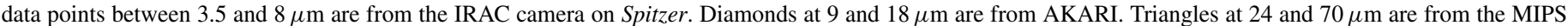
camera on Spitzer. Circles between 12 and $100 \mu \mathrm{m}$ are from IRAS. The IRS spectrum from Spitzer is plotted at mid-IR wavelengths.

(A color version of this figure is available in the online journal.) 

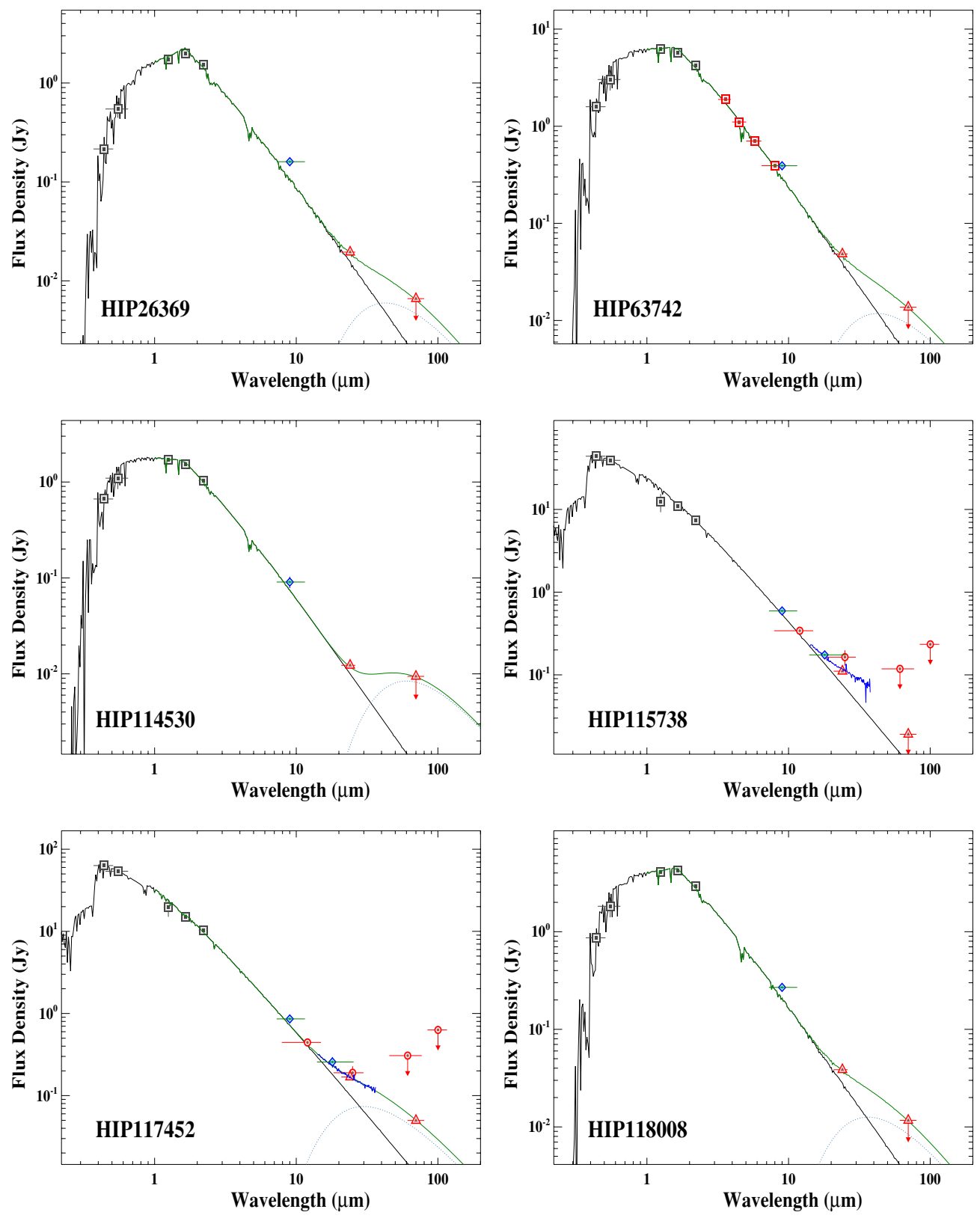

Figure 2. SEDs for AB Dor stars in Table 8 with probable or definite infrared excess emission.

(A color version of this figure is available in the online journal.)

sample, conservatively, we ignore this possibility and treat the $3 \%$ as an effective error in the photospheric model predictions. Therefore, in Tables 8-10 the listed 24 and $70 \mu \mathrm{m}$ photospheric fluxes are those predicted from the theoretical atmospheric model but multiplied by a factor of 1.03. In addition to this small mean offset, the Figure 8 histogram indicates a $\pm 7 \%$ uncertainty in the individual $24 \mu \mathrm{m}$ flux densities due to some combination of model uncertainties, measurement error, and perhaps other factors.

The MIPS Handbook lists $4 \%$ as the systematic $24 \mu \mathrm{m}$ absolute flux calibration uncertainty (see also Engelbracht et al. 2007). This $4 \%$ is negligible when added in quadrature with the measurement uncertainty estimated just below.

We checked the 7\% flux density uncertainty deduced from Figure 8 in the following fashion. For stars in Tables 8-10 with measured $24 \mu \mathrm{m}$ flux density less than the model photo-
Table 7

Spitzer Observational Programs That Include Nearby Argus Stars

\begin{tabular}{ccclcc}
\hline \hline Star & Spitzer & AORKEY & \multicolumn{1}{c}{ Date of } & \multicolumn{2}{c}{ Integ. Time (s) } \\
\cline { 5 - 6 } & Program ID & Archive ID & Observation & $24 \mu \mathrm{m}$ & $70 \mu \mathrm{m}$ \\
\hline HD 84075 & 72 & 4545280 & 2004 Feb 23 & 288 & 630 \\
HIP 50191 & 10 & 3707136 & 2005 May 18 & 48 & 126 \\
CD-74 673 & 148 & 5355520 & 2004 Aug 23 & 288 & 756 \\
HIP 68994 & 20597 & 15591424 & 2006 Apr 8 & 30 & 15 \\
HIP 79797 & 10 & 3720448 & 2004 Mar 17 & 48 & 126 \\
HIP 98495 & 10 & 3725824 & 2004 Apr 8 & 48 & 126 \\
HIP 99770 & 10 & 3726848 & 2004 Oct 16 & 48 & 126 \\
\hline
\end{tabular}

Note. The infrared spectrum of HIP $57632(=\beta$ Leo, Table 4$)$ has been extensively studied (beginning with IRAS; Rhee et al. 2007b) and we therefore have not included this star in Table 7. 
Table 8

AB Dor Stars: MIPS Flux Densities

\begin{tabular}{|c|c|c|c|c|c|c|c|c|c|c|c|}
\hline \multirow{3}{*}{$\begin{array}{r}\text { Star } \\
\text { PW And }\end{array}$} & \multirow{2}{*}{\multicolumn{2}{|c|}{$\frac{\text { Photosph. Meas. }}{24 \mu \mathrm{m}(\mathrm{mJy})}$}} & \multirow{3}{*}{$\begin{array}{c}\text { Excess? } \\
\mathrm{N}\end{array}$} & \multirow{2}{*}{\multicolumn{2}{|c|}{$\frac{\text { Photosph. Meas. }}{70 \mu \mathrm{m}(\mathrm{mJy})}$}} & \multirow{3}{*}{$\begin{array}{l}\text { Excess? } \\
\mathrm{N}\end{array}$} & \multirow[t]{3}{*}{ IRS? } & \multirow{3}{*}{$\begin{array}{c}T_{\text {star }} \\
(\mathrm{K}) \\
4700\end{array}$} & \multirow{3}{*}{$\begin{array}{c}R_{\text {star }} \\
\left(R_{\odot}\right) \\
0.54\end{array}$} & \multirow{3}{*}{$\begin{array}{c}T_{\text {dust }} \\
(\mathrm{K}) \\
\end{array}$} & \multirow{3}{*}{$\begin{array}{l}L_{\text {dust }} / L \\
\left(\times 10^{-5}\right)\end{array}$} \\
\hline & & & & & & & & & & & \\
\hline & 20.6 & 21.8 & & 2.3 & & & & & & & \\
\hline HIP 3589 & 19.9 & 21.9 & $\mathrm{~N}$ & 2.2 & & $\mathrm{~N}$ & & 6000 & 1.19 & & \\
\hline HIP 5191 & 8.4 & 8.62 & $\mathrm{~N}$ & 0.9 & & $\mathrm{~N}$ & & 4900 & 0.87 & & \\
\hline HIP 6276 & 16.3 & 19.8 & $\mathrm{Y}$ & 1.8 & & $\mathrm{~N}$ & $\mathrm{Y}$ & 5200 & 0.84 & 135 & 5.2 \\
\hline HIP 10272 & 35.7 & 37.0 & $\mathrm{~N}$ & 3.9 & & $\mathrm{~N}$ & & 5200 & 1.14 & & \\
\hline HIP 13027A & 46.0 & 26.7 & $\mathrm{~N}$ & 5.0 & & $\mathrm{~N}$ & & 5200 & 1.31 & & \\
\hline HIP 13027B & 39.2 & 26.7 & $\mathrm{~N}$ & 4.3 & & $\mathrm{~N}$ & & 5000 & 1.23 & & \\
\hline IS Eri & 13.7 & 18.8 & $\mathrm{Y}$ & 1.5 & & $\mathrm{~N}$ & $\mathrm{Y}$ & 5200 & 0.88 & 150 & 9.9 \\
\hline HIP 14807 & 6.2 & 7.57 & $\mathrm{Y}$ & 0.7 & & $\mathrm{~N}$ & & 4600 & 0.79 & $>70$ & $<37$ \\
\hline HIP 14809 & 11.5 & 16.1 & $\mathrm{Y}$ & 1.3 & & $\mathrm{~N}$ & & 5900 & 0.91 & $>97$ & $<10$ \\
\hline HIP 15353 & 37.1 & 35.8 & $\mathrm{~N}$ & 4.3 & & $\mathrm{~N}$ & & 8100 & 1.62 & & \\
\hline HIP $16563 \mathrm{~A}$ & 19.1 & 25.0 & $Y ?^{\mathrm{a}}$ & 2.1 & & $\mathrm{~N}$ & & 5400 & 0.86 & $>115$ & $<7.9$ \\
\hline HIP 16563B & 6.6 & 9.31 & $Y ?^{\mathrm{a}}$ & 0.7 & & $\mathrm{~N}$ & & 4400 & 0.54 & $>93$ & $<32$ \\
\hline HIP 17695 & 19.6 & 17.6 & $\mathrm{~N}$ & 2.3 & & $\mathrm{~N}$ & & 3400 & 0.49 & & \\
\hline HIP 18859 & 154 & 210 & $\mathrm{Y}$ & 18.0 & 323 & $\mathrm{Y}$ & $\mathrm{Y}$ & 6200 & 1.24 & 70 & 11 \\
\hline HIP 19183 & 16.1 & 14.5 & $\mathrm{~N}$ & 1.9 & & $\mathrm{~N}$ & $\mathrm{Y}$ & 6400 & 1.13 & & \\
\hline HIP 25283 & 37.5 & 35.3 & $\mathrm{~N}$ & 4.3 & 38.3 & $\mathrm{Y}$ & & 4000 & 0.70 & 60 & 17.5 \\
\hline AB DorA & 101 & 111 & $\mathrm{~N}$ & 11.1 & & $\mathrm{~N}$ & & 4800 & 0.90 & & \\
\hline AB DorB & 7.2 & 7.01 & $\mathrm{~N}$ & 0.8 & & $\mathrm{~N}$ & & & & & \\
\hline HIP 26369 & 16.0 & 19.5 & $\mathrm{Y}$ & 1.8 & & $\mathrm{~N}$ & & 4500 & 0.60 & $>120$ & $<9.5$ \\
\hline HIP 26373 & 31.5 & 33.3 & $\mathrm{~N}$ & 3.4 & & $\mathrm{~N}$ & $\mathrm{Y}$ & 5200 & 0.79 & & \\
\hline CD-35 2722 & 16.6 & 14.4 & $\mathrm{~N}$ & 2.0 & & $\mathrm{~N}$ & & 3600 & 0.65 & & \\
\hline HIP 30314 & 63.3 & 70.9 & $\mathrm{~N}$ & 6.9 & & $\mathrm{~N}$ & $\mathrm{Y}$ & 6000 & 1.02 & & \\
\hline GSC8894-0426 & 17.6 & 13.6 & $\mathrm{~N}$ & 2.1 & & $\mathrm{~N}$ & & 3200 & 0.58 & & \\
\hline HIP 31711 & 108 & 117 & $\mathrm{~N}$ & 11.6 & & $\mathrm{~N}$ & & 5700 & 1.27 & & \\
\hline HIP 31878 & 19.2 & 20.2 & $\mathrm{~N}$ & 2.2 & & $\mathrm{~N}$ & & 4300 & 0.60 & & \\
\hline HIP 36349 & 65.0 & 49.8 & $\mathrm{~N}$ & 7.7 & & $\mathrm{~N}$ & & 3400 & 0.85 & & \\
\hline HIP 51317 & 86.3 & 73.7 & $\mathrm{~N}$ & 10.1 & & $\mathrm{~N}$ & & 3500 & 0.45 & & \\
\hline $\mathrm{BD}+201790$ & 13.1 & 12.2 & $\mathrm{~N}$ & 1.5 & & $\mathrm{~N}$ & $\mathrm{Y}$ & 4400 & 0.58 & & \\
\hline HIP 63742 & 41.6 & 48.4 & $\mathrm{Y}$ & 4.4 & & $\mathrm{~N}$ & & 5200 & 0.83 & $>120$ & $<4.5$ \\
\hline HIP 76768 & 13.7 & 13.6 & $\mathrm{~N}$ & 1.6 & & $\mathrm{~N}$ & & 4100 & 1.00 & & \\
\hline HIP 81084 & 9.9 & 8.15 & $\mathrm{~N}$ & 1.2 & & $\mathrm{~N}$ & & 3700 & 0.66 & & \\
\hline HIP 82688 & 19.2 & 20.9 & $\mathrm{~N}$ & 2.1 & & $\mathrm{~N}$ & $\mathrm{Y}$ & 6000 & 1.14 & & \\
\hline HD 317617 & 6.2 & 5.49 & $\mathrm{~N}$ & 0.7 & & $\mathrm{~N}$ & & 4700 & 0.83 & & \\
\hline HIP 86346 & 15.0 & 16.7 & $\mathrm{~N}$ & 1.7 & & $\mathrm{~N}$ & & 4200 & 0.60 & & \\
\hline HIP 93580 & 52.6 & 55.7 & $\mathrm{~N}$ & 6.1 & & $\mathrm{~N}$ & & 7700 & 1.88 & & \\
\hline HIP 106231 & 21.5 & 23.5 & $\mathrm{~N}$ & 2.4 & & $\mathrm{~N}$ & & 4500 & 0.71 & & \\
\hline HIP 107948 & 14.7 & 12.6 & $\mathrm{~N}$ & 1.7 & & $\mathrm{~N}$ & & 3300 & 0.84 & & \\
\hline HIP 109268 & 951 & 970 & $\mathrm{~N}$ & 104 & & $\mathrm{~N}$ & & 12500 & 3.77 & & \\
\hline HIP 110526 & 48.8 & 40.9 & $\mathrm{~N}$ & 5.8 & & $\mathrm{~N}$ & & 3200 & 0.78 & & \\
\hline HIP 113579 & 29.0 & 31.4 & $\mathrm{~N}$ & 3.2 & & $\mathrm{~N}$ & $\mathrm{Y}$ & 5900 & 0.94 & & \\
\hline HIP 113597 & 31.2 & 26.2 & $\mathrm{~N}$ & 3.6 & & $\mathrm{~N}$ & & 3800 & 1.09 & & \\
\hline HIP 114066 & 12.3 & 11.1 & $\mathrm{~N}$ & 1.4 & & $\mathrm{~N}$ & & 4100 & 0.56 & & \\
\hline HIP 114530 & 10.3 & 12.3 & $\mathrm{Y}$ & 1.1 & & $\mathrm{~N}$ & & 5600 & 0.92 & $>85$ & $<7.2$ \\
\hline HIP $115738^{a}$ & 78.2 & 111 & $\mathrm{Y}$ & 8.9 & & $\mathrm{~N}$ & $\mathrm{Y}$ & 9000 & 1.93 & & \\
\hline HIP 117452 & 104 & 168 & $\mathrm{Y}$ & 11.8 & 49.7 & $\mathrm{Y}$ & $\mathrm{Y}$ & 9200 & 1.96 & 170 & 2.9 \\
\hline HIP 118008 & 28.9 & 38.6 & $\mathrm{Y}$ & 3.2 & & $\mathrm{~N}$ & & 5000 & 0.71 & $>140$ & $<8.9$ \\
\hline
\end{tabular}

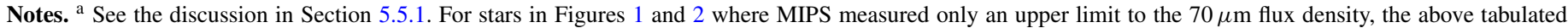

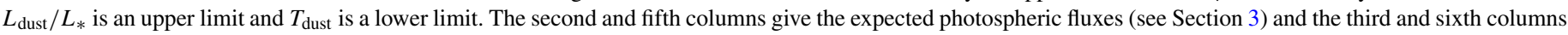
give the MIPS measured fluxes.

sphere (corrected upward by the factor of 1.03 mentioned just above), we calculate a percentage deficit given by the ratio of photosphere to measured $24 \mu \mathrm{m}$ flux density and then minus unity. For K8 to M3 stars in the AB Dor group (Tables 5 and 8), this flux deficit often falls between $14 \%$ and $33 \%$, but for earliertype AB Dor stars, the largest flux deficits are more modest; only HIP 19183 (14\%) and HD 317617 (15.5\%) have flux deficits $>11 \%$. (HD 317617 is the faintest $24 \mu \mathrm{m}$ star in Table 8.) In Table 9 (Tuc/Hor and Columba stars), the only non-M-type stars with flux deficits $>11 \%$ are HIP 2484 (14\%), HIP 2487
(15.5\%), and CD-34 2406 (23\%). (The latter star is by far the faintest $24 \mu \mathrm{m}$ star listed in Table 9.)

The preceding paragraph indicates that while $\pm 11 \%$ appears to be an appropriate $24 \mu \mathrm{m}$ flux density uncertainty for most non-M-type stars in Tables 8-10, adaptation of $16 \%$ for this uncertainty should encompass just about all such stars. This choice also encompasses the $7 \%$ flux uncertainty at $24 \mu \mathrm{m}$ for stars in Figure 8, as mentioned above.

Thus, when a MIPS measured $24 \mu \mathrm{m}$ flux is a factor 1.16 larger than a photospheric flux listed in Tables 8-10, we deem 
Table 9

Tuc/Hor \& Columba Stars: MIPS Flux Densities

\begin{tabular}{|c|c|c|c|c|c|c|c|c|c|c|c|}
\hline Star & $\frac{\text { Photc }}{24}$ & $\frac{\text { Meas. }}{\text { hy) }}$ & Excess? & $\frac{\text { Phot }}{70}$ & $\frac{\text { Meas. }}{\text { Jy) }}$ & Excess? & IRS? & $\begin{array}{l}T_{\text {star }} \\
(\mathrm{K})\end{array}$ & $\begin{array}{l}R_{\text {star }} \\
\left(R_{\odot}\right)\end{array}$ & $\begin{array}{c}T_{\text {dust }} \\
(\mathrm{K})\end{array}$ & $\begin{array}{c}L_{\text {dust }} / L_{*} \\
\left(\times 10^{-5}\right)\end{array}$ \\
\hline HIP 490 & 25.3 & 27.5 & $\mathrm{~N}$ & 3.0 & 162 & $\mathrm{Y}$ & $\mathrm{Y}$ & 6100 & 1.06 & 50 & 34.0 \\
\hline HIP 1113 & 10.8 & 12.7 & $\mathrm{Y}$ & 1.1 & 18.7 & $\mathrm{Y}$ & & 5600 & 0.82 & 70 & 11.9 \\
\hline HIP 1481 & 24.9 & 35.2 & $\mathrm{Y}$ & 2.9 & 14.3 & $\mathrm{Y}$ & $\mathrm{Y}$ & 6200 & 1.06 & 132 & 7.1 \\
\hline HIP 1910 & 9.8 & 9.03 & $\mathrm{~N}$ & 1.1 & & $\mathrm{~N}$ & & 3800 & 0.94 & & \\
\hline HIP 1993 & 8.2 & 7.68 & $\mathrm{~N}$ & 0.96 & & $\mathrm{~N}$ & & 3700 & 0.70 & & \\
\hline HIP 2484 & 97.3 & 87.8 & $\mathrm{~N}$ & 10.4 & & $\mathrm{~N}$ & $\mathrm{Y}$ & 11500 & 1.72 & & \\
\hline HIP 2487 & 158 & 140 & $\mathrm{~N}$ & 18.3 & & $\mathrm{~N}$ & & 8200 & & & \\
\hline HIP 2578 & 68.3 & 231 & $\mathrm{Y}$ & 7.7 & 59.9 & $\mathrm{Y}$ & $\mathrm{Y}$ & 9300 & 1.67 & 200 & 9.4 \\
\hline HIP 2729 & 18.1 & 17.1 & $\mathrm{~N}$ & 2.1 & & $\mathrm{~N}$ & & 4400 & 1.21 & & \\
\hline HIP 3556 & 9.6 & 7.71 & $\mathrm{~N}$ & 1.1 & & $\mathrm{~N}$ & & 3500 & 0.80 & & \\
\hline CPD-64 120 & 4.1 & 5.28 & $\mathrm{Y}$ & 0.44 & & & & 5200 & 0.88 & & \\
\hline HD 8558 & 12.0 & 14.3 & $\mathrm{Y}$ & 1.3 & & $\mathrm{~N}$ & & 5800 & 0.95 & $>75$ & $<9.0$ \\
\hline HD 9054 & 12.7 & 14.1 & $\mathrm{~N}$ & 1.4 & & $\mathrm{~N}$ & & 4900 & 0.80 & & \\
\hline HIP 9141 & 17.3 & 24.7 & $\mathrm{Y}$ & 1.9 & & $\mathrm{~N}$ & $\mathrm{Y}$ & 5800 & 0.98 & 160 & 7.9 \\
\hline HD 12894 & 47.0 & 48.6 & $\mathrm{~N}$ & 5.5 & & $\mathrm{~N}$ & & 6800 & 1.61 & & \\
\hline HD 13183 & 12.5 & 14.1 & $\mathrm{Y} ?$ & 1.35 & & $\mathrm{~N}$ & & 5400 & 1.03 & & \\
\hline HD 13246 & 23.7 & 47.3 & $\mathrm{Y}$ & 2.76 & 31.7 & $\mathrm{Y}$ & $\mathrm{Y}$ & 6200 & 1.13 & 125 & 17.0 \\
\hline CD-60 416 & 6.8 & 8.30 & $\mathrm{~N}$ & 0.76 & & $\mathrm{~N}$ & $\mathrm{Y}$ & 4600 & 0.77 & & \\
\hline HD 14228 & 176 & 188 & $\mathrm{~N}$ & 19.1 & & $\mathrm{~N}$ & & 12000 & 2.53 & & \\
\hline HIP 12394 & 129 & 132 & $\mathrm{~N}$ & 14.0 & & $\mathrm{~N}$ & & 10500 & 2.23 & & \\
\hline HIP 12413 & 114 & 117 & $\mathrm{~N}$ & 13.2 & & $\mathrm{~N}$ & & 8300 & 1.93 & & \\
\hline CD-53 544 & 15.1 & 15.0 & $\mathrm{~N}$ & 1.7 & & $\mathrm{~N}$ & & 4200 & 0.74 & & \\
\hline AF Hor & 9.77 & 7.94 & $\mathrm{~N}$ & 1.14 & & $\mathrm{~N}$ & & 3500 & 0.57 & & \\
\hline CD-58 553 & 5.7 & 5.35 & $\mathrm{~N}$ & 0.65 & & $\mathrm{~N}$ & & 4400 & 0.74 & & \\
\hline HIP 14451 & 34.0 & 34.2 & $\mathrm{~N}$ & 3.9 & & $\mathrm{~N}$ & & 8000 & 1.54 & & \\
\hline HIP 15247 & 25.4 & 27.5 & $\mathrm{~N}$ & 3.0 & & $\mathrm{~N}$ & & 6200 & 1.10 & & \\
\hline CD-46 1064 & 10.2 & 11.8 & Y? & 1.1 & & $\mathrm{~N}$ & & 4800 & 0.95 & & \\
\hline HD 21997 & 27.0 & 54.6 & $\mathrm{Y}$ & 2.9 & 70.5 & $\mathrm{Y}$ & $\mathrm{Y}$ & 8200 & 1.74 & 60 & 57.6 \\
\hline HIP 16853 & 23.3 & 27.2 & Y & 2.5 & & $\mathrm{~N}$ & & 6000 & 1.10 & $>95$ & $<2.8$ \\
\hline HIP 17764 & 24.8 & 42.6 & $\mathrm{Y}$ & 2.9 & 36.0 & $\mathrm{Y}$ & $\mathrm{Y}$ & 6700 & 1.37 & 110 & 11.4 \\
\hline BD-15 705 & 7.3 & 7.43 & $\mathrm{~N}$ & 0.83 & & $\mathrm{~N}$ & & 4600 & 1.05 & & \\
\hline HIP 21632 & 12.2 & 12.5 & $\mathrm{~N}$ & 1.3 & & $\mathrm{~N}$ & & 5900 & 1.03 & & \\
\hline HIP 21965 & 28.1 & 29.7 & $\mathrm{~N}$ & 3.3 & 25.4 & $\mathrm{Y}$ & & 6600 & 1.55 & 80 & 4.4 \\
\hline HIP 22295 & 12.6 & 16.4 & $\mathrm{Y}$ & 1.5 & 11.3 & $\mathrm{Y}$ & & 6300 & 1.10 & 100 & 7.2 \\
\hline HIP $23179^{a}$ & $? ?$ & 92.6 & $\mathrm{~N}$ & $\sim 10$ & & $\mathrm{~N}$ & $\mathrm{Y}$ & 8000 & 2.30 & & \\
\hline HIP 24947 & 25.4 & 32.6 & $\mathrm{Y}$ & 2.9 & 11.0 & $\mathrm{Y}$ & & 6300 & 1.18 & 135 & 4.9 \\
\hline HD 36869 & 13.6 & 14.0 & $\mathrm{~N}$ & 1.5 & & $\mathrm{~N}$ & & 5800 & 1.21 & & \\
\hline HIP 26309 & 31.3 & 67.9 & Y & 3.6 & & $\mathrm{~N}$ & $\mathrm{Y}$ & 8000 & 1.46 & 160 & 8.2 \\
\hline AT Col & 7.7 & 9.54 & $\mathrm{Y}$ & 0.84 & & $\mathrm{~N}$ & & 5100 & 0.95 & & \\
\hline HIP 26453 & 22.3 & 56.1 & $\mathrm{Y}$ & 2.6 & 116 & $\mathrm{Y}$ & $\mathrm{Y}$ & 6700 & 1.40 & 90 & 32.5 \\
\hline CD-34 2406 & 2.05 & 1.70 & $\mathrm{~N}$ & 0.23 & & $\mathrm{~N}$ & & 4800 & 0.52 & & \\
\hline HD 38206 & 32.5 & 114 & Y & 3.6 & 388 & $\mathrm{Y}$ & $\mathrm{Y}$ & 9900 & 1.68 & 85 & 19.1 \\
\hline HIP 28036 & 23.8 & 23.7 & $\mathrm{~N}$ & 2.8 & & $\mathrm{~N}$ & & 6300 & 1.36 & & \\
\hline HIP 30030 & 17.3 & 20.4 & Y & 2.0 & & $\mathrm{~N}$ & $\mathrm{Y}$ & 6100 & 1.08 & 160 & 3.2 \\
\hline HIP $30034^{\mathrm{b}}$ & 10.4 & 13.1 & $\mathrm{Y}$ & 1.1 & & $\mathrm{~N}$ & & 5200 & 0.87 & & \\
\hline HIP 32104 & 67.4 & 66.7 & $\mathrm{~N}$ & 7.8 & & $\mathrm{~N}$ & & 8600 & 1.57 & & \\
\hline HIP 32235 & 7.9 & 9.81 & $\mathrm{Y}$ & 0.86 & & $\mathrm{~N}$ & & 5800 & 0.89 & $>75$ & $<13.2$ \\
\hline HIP $32435^{b}$ & 21.5 & 33.2 & $\mathrm{Y}$ & 2.5 & & & $\mathrm{Y}$ & 6500 & 1.35 & 140 & 7.4 \\
\hline HIP 33737 & 5.9 & 6.29 & $\mathrm{~N}$ & 0.66 & & $\mathrm{~N}$ & & 4900 & 0.94 & & \\
\hline HIP 83494 & 41.5 & 47.3 & $Y ?$ & 4.8 & & $\mathrm{~N}$ & & 7700 & 1.63 & & \\
\hline HIP 84642 & 6.75 & 7.87 & $\mathrm{Y}$ & 0.73 & & $\mathrm{~N}$ & & 5200 & 0.84 & & \\
\hline HIP 100751 & 680 & 656 & $\mathrm{~N}$ & 78.4 & & $\mathrm{~N}$ & & 15000 & 5.25 & & \\
\hline HIP 104308 & 26.4 & 25.7 & $\mathrm{~N}$ & 3.1 & & $\mathrm{~N}$ & & 7600 & 1.61 & & \\
\hline HIP 105388 & 11.1 & 20.1 & $\mathrm{Y}$ & 1.2 & 36.3 & $\mathrm{Y}$ & $\mathrm{Y}$ & 5700 & 0.86 & 86 & 27.7 \\
\hline HIP 105404 & 15.2 & 19.5 & $\mathrm{Y}$ & 1.7 & & $\mathrm{~N}$ & & 5100 & 1.07 & $>120$ & $<8.6$ \\
\hline HIP 107345 & 6.5 & 6.44 & $\mathrm{~N}$ & 0.76 & & $\mathrm{~N}$ & & 3900 & 0.69 & & \\
\hline HIP 107947 & 27.2 & 31.0 & $Y ?$ & 3.2 & 9.6 & $\mathrm{Y}$ & & 6400 & 1.20 & 110 & 2.4 \\
\hline HIP 108195 & 80.5 & 77.3 & $\mathrm{~N}$ & 9.4 & & $\mathrm{~N}$ & & 6600 & 2.1 & & \\
\hline HIP 108422 & 13.2 & 17.3 & $\mathrm{Y}$ & 1.4 & & $\mathrm{~N}$ & & 5200 & 1.18 & $>100$ & $<12$ \\
\hline HIP $116748^{a}$ & 25.0 & 24.8 & $\mathrm{~N}$ & 2.7 & & $\mathrm{~N}$ & & 5200 & 1.37 & & \\
\hline HIP 118121 & 78.7 & 77.8 & $\mathrm{~N}$ & 9.0 & & $\mathrm{~N}$ & & 9000 & 1.90 & & \\
\hline
\end{tabular}

Notes.

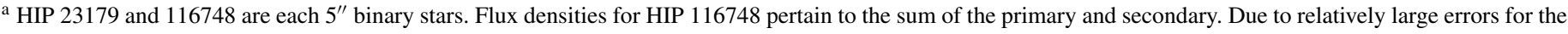

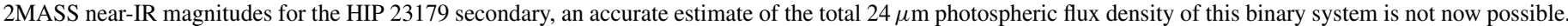

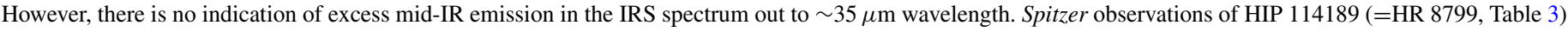

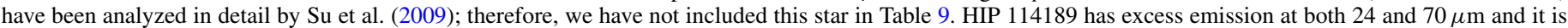

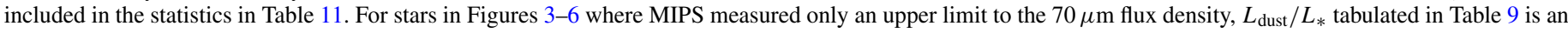
upper limit and $T_{\text {dust }}$ is a lower limit.

$\mathrm{b}$ See the discussion of HIP 30034 and HIP 32435 in Section 5.5.1. 
Table 10

Argus Stars: MIPS Flux Densities

\begin{tabular}{|c|c|c|c|c|c|c|c|c|c|c|c|}
\hline \multirow[t]{2}{*}{ Star } & Phot & Meas. & \multirow[t]{2}{*}{ Excess? } & Phot & Meas. & \multirow[t]{2}{*}{ Excess? } & \multirow[t]{2}{*}{ IRS? } & \multirow{2}{*}{$\begin{array}{c}T_{\text {star }} \\
(\mathrm{K})\end{array}$} & \multirow{2}{*}{$\begin{array}{l}R_{\text {star }} \\
\left(R_{\odot}\right)\end{array}$} & \multirow{2}{*}{$\begin{array}{r}T_{\text {dust }} \\
(\mathrm{K}) \\
\end{array}$} & \multirow{2}{*}{$\begin{array}{l}L_{\text {dust }} / L_{*} \\
\left(\times 10^{-5}\right)\end{array}$} \\
\hline & \multicolumn{2}{|c|}{$24 \mu \mathrm{m}$ (mJy) } & & \multicolumn{2}{|c|}{$70 \mu \mathrm{m}$ (mJy) } & & & & & & \\
\hline HD 84075 & 9.0 & 12.6 & $\mathrm{Y}$ & 1.0 & 35.8 & $\mathrm{Y}$ & $\mathrm{Y}$ & 6000 & 1.03 & 170 & 25.1 \\
\hline HIP 50191 & 215 & 266 & $\mathrm{Y}$ & 24.4 & 46.2 & $\mathrm{Y}$ & & 9000 & 2.03 & 180 & 1.0 \\
\hline CD-74 673 & 4.6 & 4.85 & $\mathrm{~N}$ & 0.51 & & $\mathrm{~N}$ & $\mathrm{Y}$ & 4800 & 0.64 & & \\
\hline HIP $68994^{\mathrm{a}}$ & 14.8 & 24.7 & $\mathrm{Y}$ & 1.7 & & & & 6600 & 1.28 & & \\
\hline HIP 79797 & 38.4 & 52.6 & $\mathrm{Y}$ & 4.4 & 39.3 & $\mathrm{Y}$ & $\mathrm{Y}$ & 8200 & 1.56 & 220 & 5.1 \\
\hline HIP 98495 & 175 & 173 & $\mathrm{~N}$ & 19.7 & 57.8 & $\mathrm{Y}$ & $\mathrm{Y}$ & 9600 & 1.85 & 90 & 0.44 \\
\hline HIP 99770 & 121 & 125 & $\mathrm{~N}$ & 13.9 & $\mathrm{~b}$ & $\mathrm{~N} ?$ & & 7600 & 2.12 & & \\
\hline
\end{tabular}

Notes.

a See the discussion of HIP 68994 in Section 5.5.1.

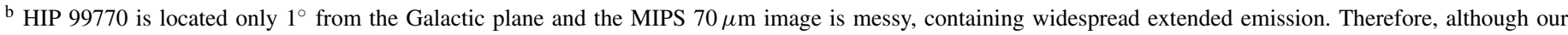

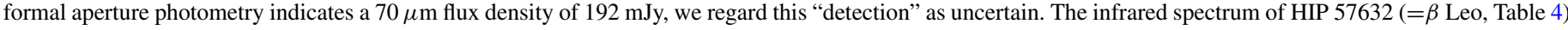

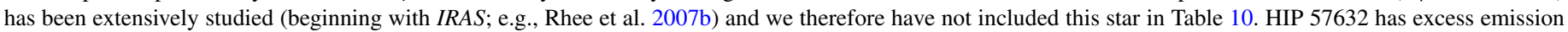
at both 24 and $70 \mu \mathrm{m}$ and it is included in the statistics in Table 11.

Table 11

Infrared Excess Fractions in Clusters/Associations

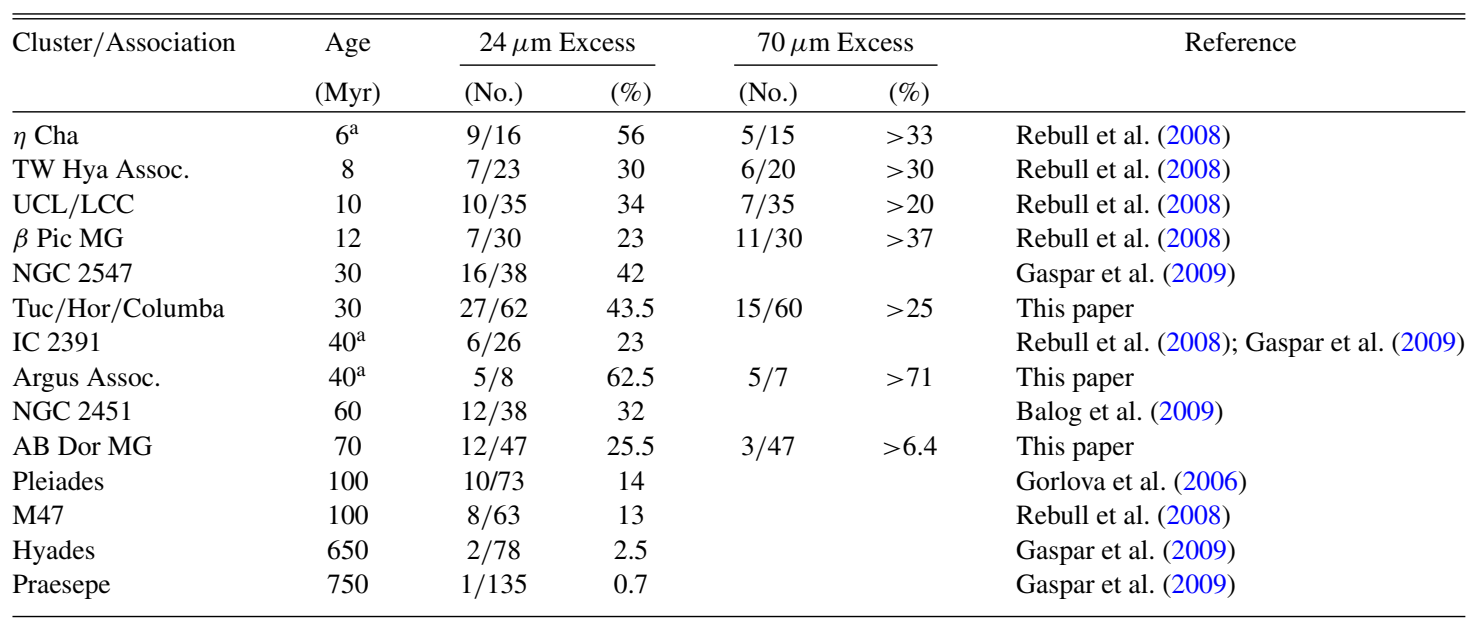

Note. ${ }^{a}$ Age from Torres et al. (2008).

a non-M-type star to have excess flux at $24 \mu \mathrm{m}$. If the apparent $24 \mu \mathrm{m}$ MIPS excess lies between $12 \%$ and $16 \%$, then in the fourth column of Tables 8-10 we enter Y? indicating possible excess emission. In fact, only three stars in Table 9 have entries of Y?, and only the binary star HIP 16563 (considered in Section 5.5.1) has a Y? entry in Table 8.

At $70 \mu \mathrm{m}$, the procedure is similar, except that we use $7 \%$ as the calibration uncertainty as recommended in the MIPS Handbook.

\section{RESULTS}

Tables 2-4 present stars we propose as new members of the AB Doradus, Tucana/Horologium, Columba, and field Argus Associations within $\sim 70$ pc of Earth. Based on their survey of southern hemisphere stars, Torres et al. (2008) introduced the Columba and Argus Associations. In addition to location in the southern hemisphere, all Columba members listed by Torres et al. lie in the R.A. range between about $2 \mathrm{hr}$ and $8 \mathrm{hr}$. In our study we have placed no a priori restriction on R.A. or decl. for stars in any moving group. Proposed membership is based on $U V W$ and standard age indicators (see below and Section 5). Future observations and traceback analysis can confirm or deny membership of each star.
According to Torres et al. (2008), the $U$ and $W$ components of Columba's space motions are more negative than those of Tuc/ Hor (see Section 5.2 and the notes to Table 3). Also, according to Table 2 of Torres et al., Columba stars are more distant, on average, than stars in Tuc/Hor. The listed $U V W$ of a few stars in our Table 3 make it difficult to place them cleanly into either Tuc/Hor or Columba. Only a few Columba stars in Table 5 of Torres et al. are as close to Earth as stars listed in our Table 3. Nine stars in our Table 3 lie in the northern hemisphere, whereas all but one of the Tuc/Hor and Columba stars in Zuckerman \& Song (2004) and Torres et al. (2008) lie in the southern hemisphere. Since Tuc/Hor and Columba have similar ages (30 Myr; Torres et al. 2008), for the purposes of understanding the evolution of dusty debris disks as a function of time, in Table 11 and Section 5.5.2 we consider members of these two associations together.

As in our previous papers on these and other young, nearby, moving groups (e.g., Song et al. 2003; Zuckerman et al. 2001a, 2001 b, 2004), we rely on a combination of techniques-including Galactic space motions $U V W$, location on color-magnitude diagrams, lithium abundance, and X-ray luminosity-to establish age and membership.

Tables 5 and 6 list Tuc/Hor and AB Dor stars observed by Spitzer as part of our GO program no. 3600 or as part of 

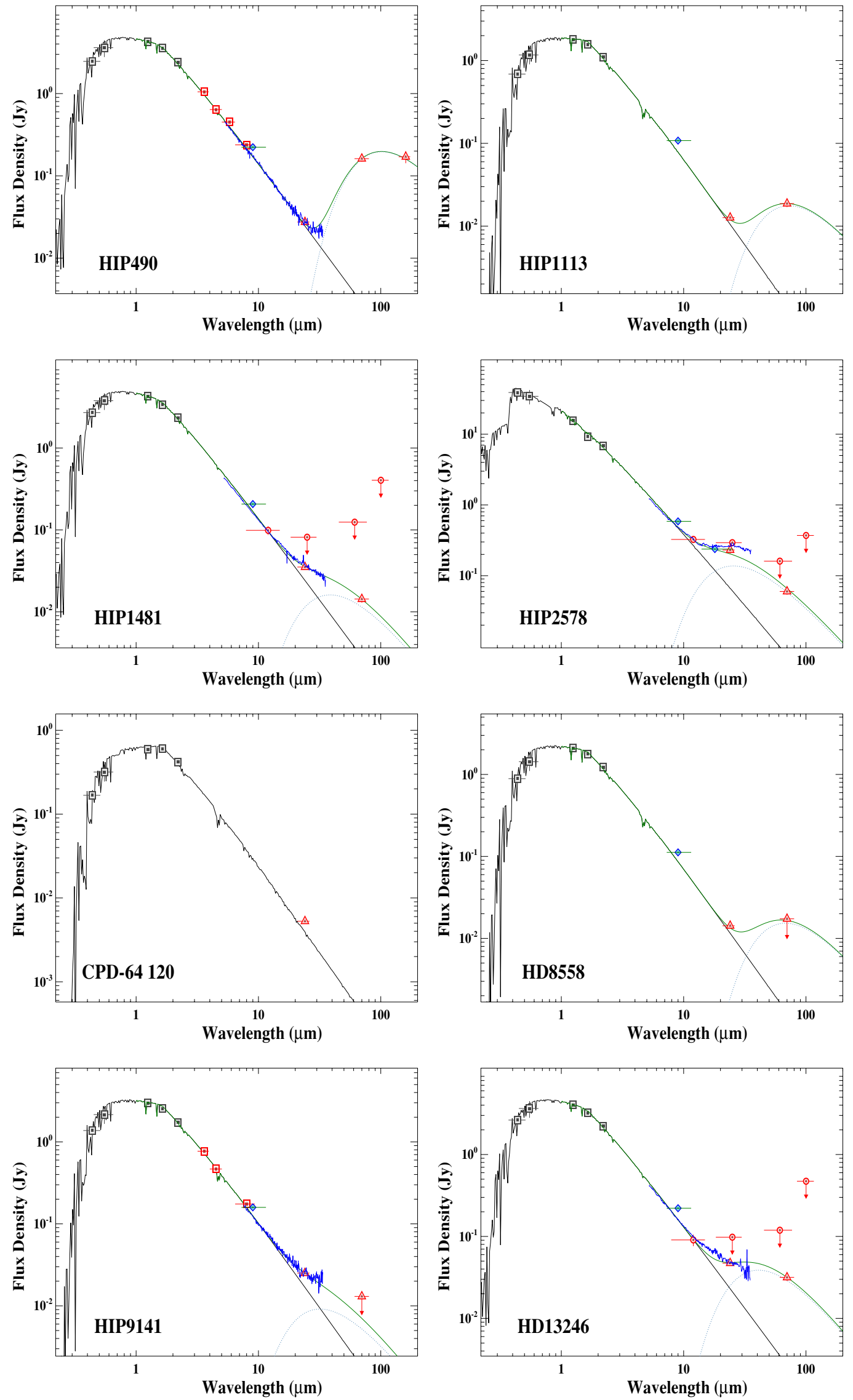

Figure 3. SEDs for Tuc/Hor stars in Table 9 with probable or definite infrared excess emission.

(A color version of this figure is available in the online journal.) 

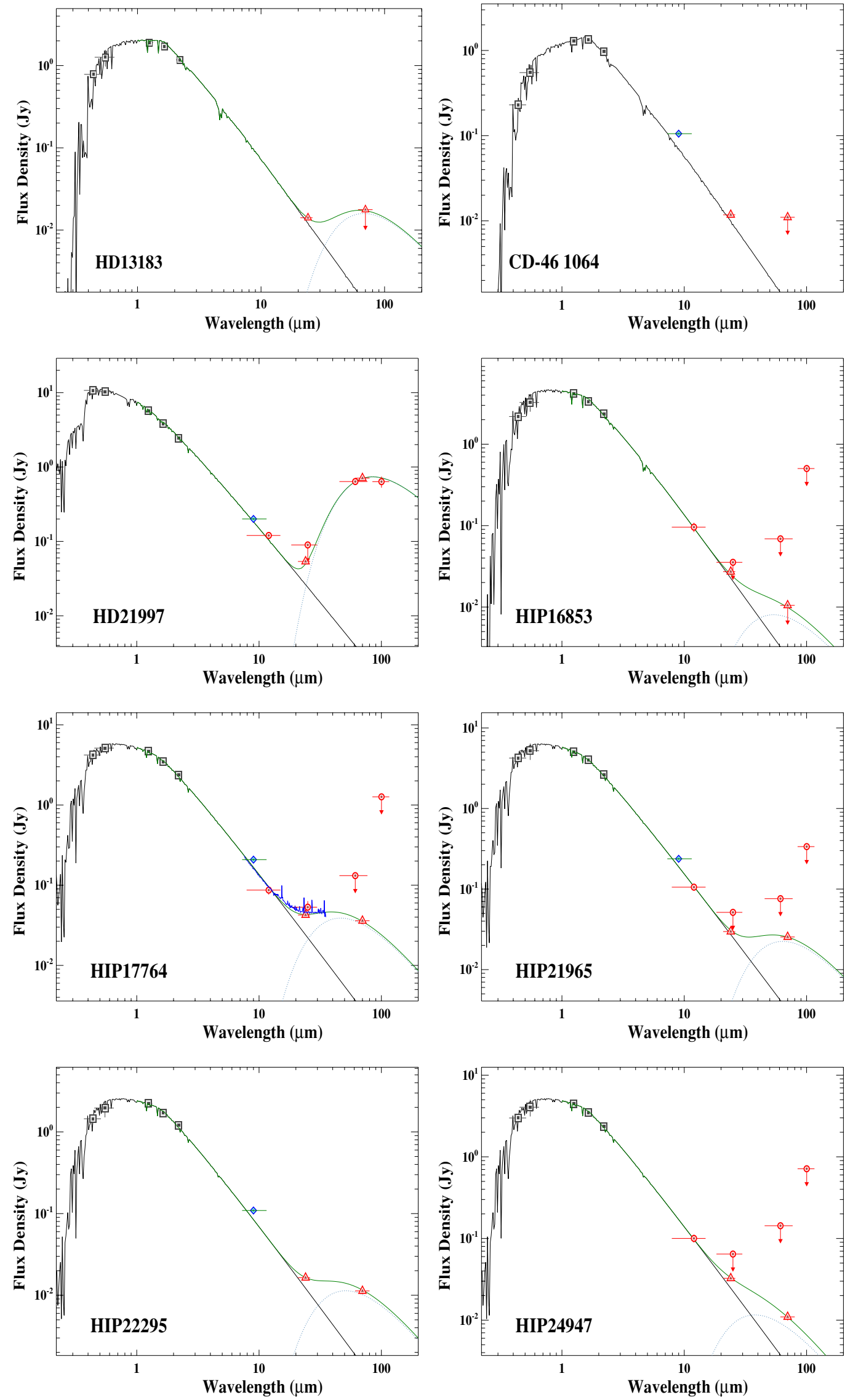

Figure 4. SEDs for Tuc/Hor stars in Table 9 with probable or definite infrared excess emission.

(A color version of this figure is available in the online journal.) 

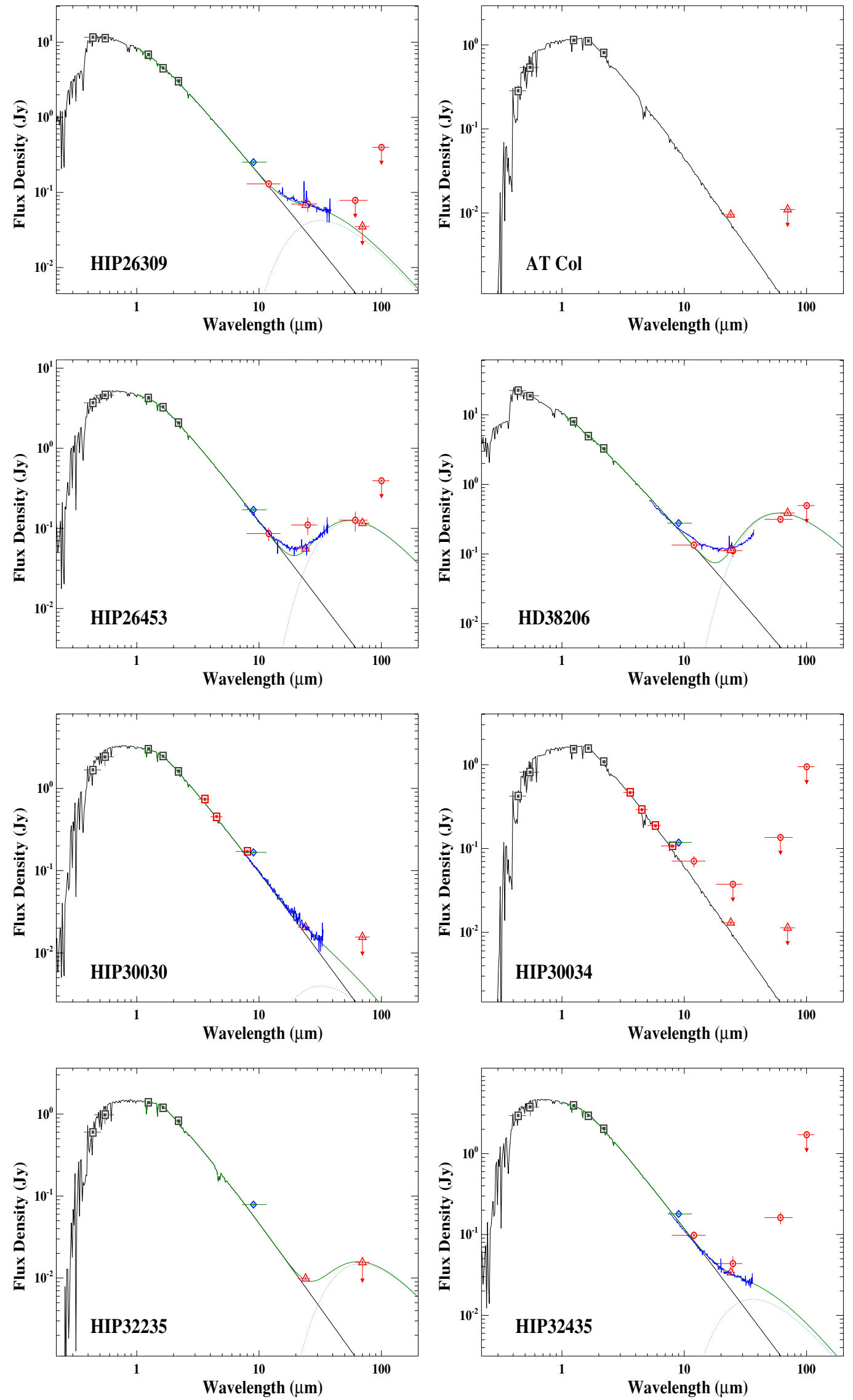

Figure 5. SEDs for Tuc/Hor stars in Table 9 with probable or definite infrared excess emission.

(A color version of this figure is available in the online journal.) 

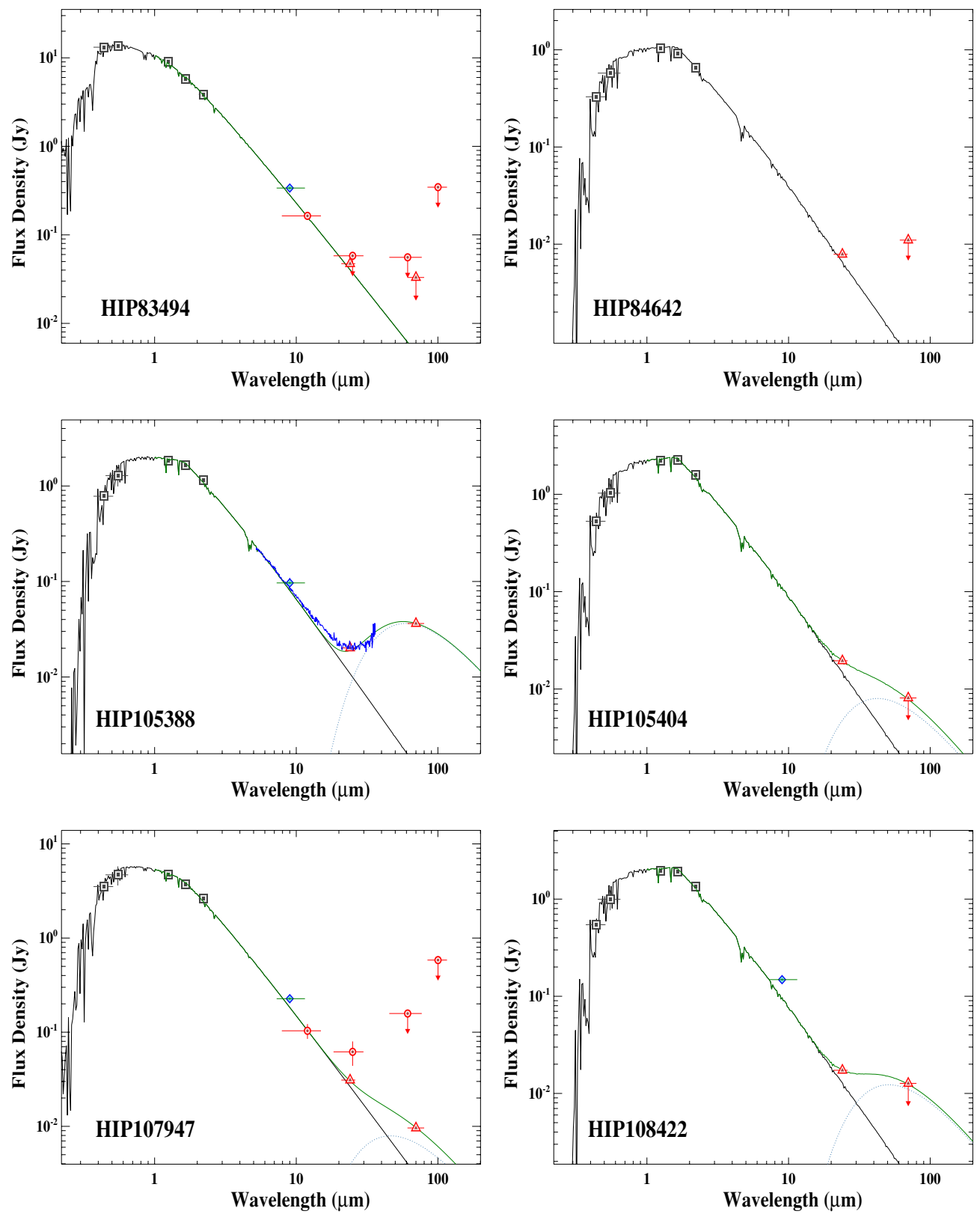

Figure 6. SEDs for Tuc/Hor stars in Table 9 with probable or definite infrared excess emission.

(A color version of this figure is available in the online journal.)

Spitzer programs by other groups; Table 7 lists these various programs for the proposed Argus Association members within $70 \mathrm{pc}$ of Earth. Tables $8-10$ present MIPS photometry while Table 11 compares the frequency of dusty debris disks in the four moving groups considered in the present paper with that of other stellar associations with previously published Spitzer results.

\section{DISCUSSION}

\subsection{AB Doradus}

While published compendia of members of the nearby Tucana/Horologium and $\beta$ Pictoris kinematic groups include stars of A-type or late-B-type or both, no stars earlier than midF spectral class are listed previously for the AB Doradus moving group (Zuckerman et al. 2004; Zuckerman \& Song 2004; Torres et al. 2008; da Silva et al. 2009). Stars proposed in Table 2 as AB
Dor members are either of A- or late-B type. Given that AB Dor stars appear to be about as numerous as Tucana and $\beta$ Pictoris stars, identification of these early-type AB Dor members while belated, is not unexpected. All Table 2 stars lie near the location of a typical Pleiades star on an A-star color-magnitude diagram (Figure 5 in Zuckerman 2001) consistent with the age of the AB Dor moving group.

HIP 22845 (HD 31295) is a well-studied $\lambda$ Boo and Spitzer and IRAS infrared excess star. Its $U V W$ of $-4.3,-23.6,-10.1$ $\pm(1.1,0.8,0.5) \mathrm{km} \mathrm{s}^{-1}$ is, at best, in marginal agreement with published $U V W$ for the AB Dor group (see the note to Table 2). HIP 22845 lies near typical Pleiades stars on an A-star color-magnitude diagram. We have not included it as a proposed AB Dor member.

HIP 117452 (Table 2) is a member of a triple system. The A0 primary is a close binary and the tertiary, HD 223340, is an early-K-type star about $75^{\prime \prime}$ to the NW. Both the (binary) 

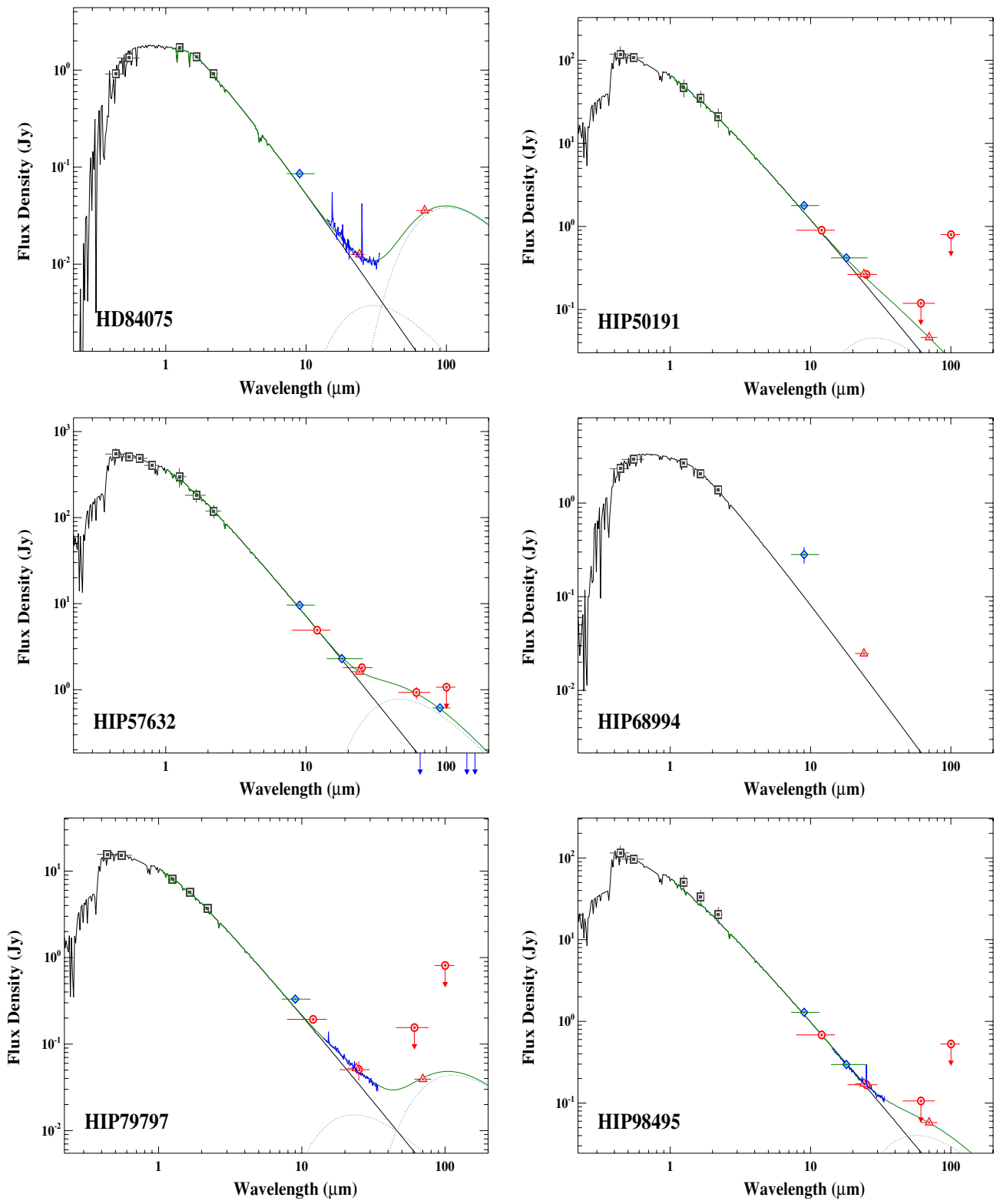

Figure 7. SEDs for Argus stars in Table 10 with probable or definite infrared excess emission.

(A color version of this figure is available in the online journal.)

primary and HD 223340 are X-ray sources. In the K star, the lithium line $\mathrm{EW}=148 \mathrm{~m} \AA$.

We regard HIP 93580 (Table 2) as only a possible member of the $\mathrm{AB}$ Dor group because the stellar $U V W$ is somewhat discordant with that of the mean $U V W$ for the group (as may be seen from the entry in Table 2 and the note to the table).

In addition to the proposed Table 2 early-type additions to the AB Dor group, our spectroscopic studies at SSO indicate that solar-type stars HD 293857, UX Col, and HD 178085 are also members (as noted independently by Torres et al. 2008 and da Silva et al. 2009).

\subsection{Tucana/Horologium and Columba}

The Tucana and Horologium Associations were proposed independently by Zuckerman \& Webb (2000) and Torres et al. (2000), respectively. Zuckerman et al. (2001b) suggested that these two moving groups are really just two adjacent regions that contain a coeval $(\sim 30$ Myr old) stream of stars with common space motion, but that the greatest concentration of stars, the "nucleus" of the overall group, is located in Tucana. Subsequently, Torres et al. (2008) introduced the notion that there are three 30 Myr old associations, Tuc/Hor, "Columba," and "Carina." As defined by Torres et al. (2008), these three differ principally in location in space-both in the plane of the sky and in their distance from Earth-and, to a lesser degree, probably in Galactic space motions $U V W$ (see also Section 4 and the note to Table 1).

Some stars in Table 3 that are likely Columba members represent a major departure from the characteristics of the Columba stars listed by Torres et al. (2008); their listed stars all fall in the R.A. range between about $2 \mathrm{hr}$ and $8 \mathrm{hr}$ and, with but one exception at $+4^{\circ}$, all have negative declinations. (The latter regularity should not come as a surprise since the Torres et al. study focused on the southern hemisphere.) Also, only 5 of the 41 Columba stars they list are within 65 pc of Earth. By contrast, all 14 of the likely Columba members of our Table 3 (based on their large negative $W$ component) are within $65 \mathrm{pc}$. 


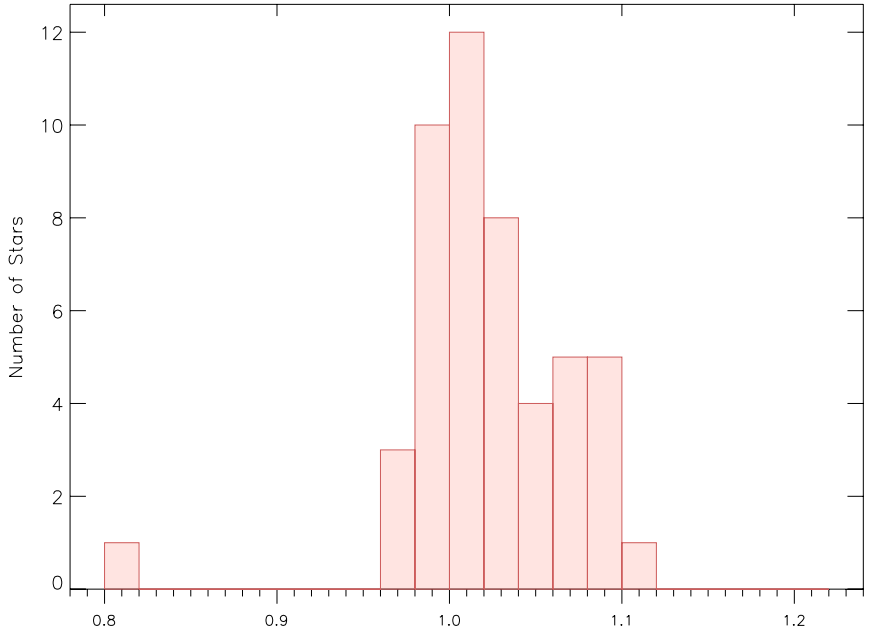

Figure 8. Histogram of the ratio of MIPS measured to model photospheric $24 \mu \mathrm{m}$ flux densities for a sample of the fainter FGK type stars from Trilling et al. (2008) without known MIPS measured excess emission. See Section 3 for details.

(A color version of this figure is available in the online journal.)

In addition, 7 of these 14 lie well outside of the plane of the sky boundaries (in R.A. and decl.) that encompass all 41 of the proposed Torres et al. members.

While the Columba Association stars proposed by Torres et al. (2008) lie primarily between about $2 \mathrm{hr}$ and $7 \mathrm{hr}$ in R.A. and in the southern hemisphere, they are only weakly constrained in distance from Earth, including even a star 189 pc away. We suggest that it would be preferable to constrain the membership to lie much closer to Earth, say out to $\sim 80 \mathrm{pc}$, and at all plane of the sky locations. An $80 \mathrm{pc}$ radius includes most of the members of the $\beta$ Pic, Tuc/Hor, and AB Dor groups as proposed by Torres et al. (2008) and by Zuckerman \& Song (2004). Based on Table 5 of Torres et al. (2008) and our Table 3, the nucleus of such a Columba moving group would lie between $5 \mathrm{hr}$ and $6 \mathrm{hr}$ R.A. and would be about $50 \mathrm{pc}$ from Earth (cf. all the stars in Table 3 from HIP 23179 to 32104 , inclusive).

The F0 star HIP 17675 (HD 23384) does not appear in Table 3 although it is young and has a $U V W$ consistent with that of Columba stars. With a radial velocity of $-1.6 \pm 0.7 \mathrm{~km} \mathrm{~s}^{-1}$ (a weighted average of our echelle measurement and that given in Gontcharov 2006), the $U V W$ of HIP 17675 is $-11.3,-21.8$, $-5.7 \pm(0.9,1.1,0.4) \mathrm{km} \mathrm{s}^{-1}$. On an A-star color-magnitude diagram (Figure 5 in Zuckerman 2001), the F0-type primary lies low thus suggesting a young age. However, our measurement of the EW of the $6708 \AA$ line of lithium is only $50 \mathrm{~m} \AA$ and the fractional X-ray luminosity, $\log \left(L_{x} / L_{\text {bol }}\right)$ based on the RASS, is about -6.0 . Together the lithium EW and the X-ray luminosity may be too small to be consistent with an age as young as 30 Myr.

Another interesting F0 star that did not find its way into Table 3 is HIP 82587 (HD 152598). The $U V W$ of $-10.7,-22.9$, $-3.6 \pm(0.4,0.5,0.5) \mathrm{km} \mathrm{s}^{-1}$ is similar to those of the Tuc/Hor and Columba Associations. The star has MIPS measured excess emission at both 24 and $70 \mu \mathrm{m}$ (Moor et al. 2009). Moor et al. give an age of $210 \pm 70 \mathrm{Myr}$ based on the star's $U V W, \mathrm{X}$-ray flux, and $6708 \AA$ lithium line strength. Our interpretation of these characteristics is consistent with an age younger than $210 \mathrm{Myr}$, although perhaps not as young as $30 \mathrm{Myr}$. Also, like HIP 17675 above, HIP 82587 lies quite low on the A-star color-magnitude diagram, suggestive of a young age. Hence, membership in the Tuc/Hor or Columba Association remains a possibility.
In addition to stars listed in Table 3, based on our independent analysis, we concur with the classification by Torres et al. (2008) of HD 38206 (HIP 26966) as a member of the Columba association. And we note that, consistent with the fact that our Table 3 stars are generally closer to Earth than the Columba stars proposed by Torres et al., HD 38206 at 69 pc from Earth is one of their nearer members.

Comments on some of the stars in Table 3 follow next. The quantity $f_{x}$ is the fractional X-ray luminosity, $\log \left(L_{x} / L_{\text {bol }}\right)$ based on the RASS. The quoted lithium line EWs are those of the $6708 \AA$ line.

HIP 12413. This is a young, multiple star system that is probably a Columba member, notwithstanding that its $W$ component of $-8.3 \pm 3.7 \mathrm{~km} \mathrm{~s}^{-1}$ may be quite different from the mean $W$ of Columba stars as estimated by Torres et al. (2008; see the note to our Table 3). On an A-star color-magnitude diagram (Zuckerman 2001) the A1-type primary is positioned in the vicinity of Pleiades stars. A ROSAT HRI image presented by Schroder \& Schmitt (2007) indicates that the primary and its M-type companion $25^{\prime \prime}$ to the north are both strong X-ray sources; no doubt the primary has a close (spatially unresolved) companion of spectral-type intermediate between it and the M-type star. The optical secondary is probably of mid-M type, although it is difficult to deduce the exact $M$ subclass because of apparent disagreement between comments in Schroder \& Schmitt (2007) and data in VizieR. In any event, the absolute $K$ mag (5.4) of the M star is such that on a color-magnitude diagram it lies well above old mid-M-type stars (e.g., Figure 2 in Zuckerman \& Song 2004) and thus is consistent with the $30 \mathrm{Myr}$ age of Columba.

HIP 14551. This is a $\sim 70^{\prime \prime}$ binary star. The M4.5 secondary (based on its $I-K=2.5$ ) is located $70^{\prime \prime}$ to the SSW of the primary. On an A-star color-magnitude diagram, the A5 primary lies near the very young star $\beta$ Pictoris. On a color-magnitude diagram, the absolute $K$ magnitude (6.3) of the secondary places it well above old, M4.5, Gliese stars (e.g., Figure 2 in Zuckerman \& Song 2004).

HIP 14913. Like HIP 12413 discussed above, HIP 14913 is a triple star system; the $\mathrm{AB}$ separation is about $00^{\prime \prime} .7$ and $\mathrm{AC}$ are separated by about $3^{\prime \prime}$.7. C is a K-type star. Concerning entries in Table 3, the lithium line EW in the F6 primary $=65 \mathrm{~m} \AA$ while $f_{x}=-4.06$ for the entire triple system considered as a single star. Our SSO spectra indicate that two nearby K-type stars CD-46 1064 (TYC 8060-1673-1) and CD-44 1173 (TYC 7574-803-1) are also Tuc/Hor members and were so identified by Torres et al. (2008). In addition, the Hipparcos measured parallax for HIP 14913 is in good agreement with the photometric parallax of the two K-type Tycho stars calculated independently by us and by Torres et al. (2008).

HIP 17248. The lithium line is too weak to be measured and $f_{x}=-3.36$; both measurements are consistent with a $30 \mathrm{Myr}$ old M0.5 star. On a color-magnitude diagram, its absolute $K$ mag (4.64) places HIP 17248 above the main sequence. Thus, all age indicators and $U V W$ are consistent with membership in the Columba Association although-because of the negative value of $W$-probably not with membership in Tuc/Hor.

HIP 17782. The star is a equal brightness 0.36 binary. The lithium and $f_{x}$ entries in Table 3 pertain to the two stars considered together.

HIP 17797. This is an $8^{\prime \prime}$ binary composed of an A1 and an A2 star. The stars lie near and below the location of a typical Pleiades star on an A-star color-magnitude diagram. The system is a weak X-ray source, suggesting the presence of a third star. 
HIP 23179. This is a $5^{\prime \prime}$ binary composed of an $\mathrm{A} 1$ and a G0 star. In the secondary the lithium line $\mathrm{EW}=165 \mathrm{m \AA}$. The system is a strong RASS X-ray source.

HIP 23362. The star lies near the location of a typical Pleiades star on an A-star color-magnitude diagram.

HD 36869. This star is AH Lep. The spectrum of the star has been measured previously by Cutispoto et al. (2002), Wichmann et al. (2003), and Lopez-Santiago et al. (2010).

HIP 26309. On an A-star color-magnitude diagram, the star lies near the very young star $\beta$ Pictoris.

HIP 26990. The spectrum of the star has been measured previously by Cutispoto et al. (2002), Wichmann et al. (2003), and Waite et al. (2005).

HIP 28474. The lithium EW and $f_{x}$ are both somewhat too small for a typical Tuc/Hor or Columba star, but because of the essentially perfect agreement of the $U V W$ of HIP 28474 with that of the mean Columba $U V W$ given in Torres et al. (2008) along with the very plausible space location of HIP 28474 with respect to other Columba stars, we deem the star to be a likely Columba member.

HIP 32104. On an A-star color-magnitude diagram, the star lies near the very young star HD 141569. HIP 32104 is a RASS source, suggesting the presence of a second star.

HIP 83494. On an A-star color-magnitude diagram, the star lies near the very young star $\beta$ Pictoris. In both R.A. and decl., HIP 83494 lies far from any Tuc/Hor or Columba star proposed by either Zuckerman \& Song (2004) or Torres et al. (2008).

HIP 84642. Chauvin et al. (2010) resolve the star as a $0^{\prime \prime} .22$ binary with delta $K_{s}=2.5 \mathrm{mag}$. As noted by Chauvin et al., probably because of the large flux contrast and small angular separation between the primary and secondary, HIP 84642 does not appear in the Hipparcos double star catalog. They deem the secondary to be of spectral type $\sim \mathrm{M} 5$ and the system age to be $\sim 40$ Myr.

The $U V W$ of HIP 84642 is in only fair agreement with the mean $U V W$ of the Tuc/Hor Association given by Zuckerman \& Song (2004) and by Torres et al. (2008) and HIP 84642 is located in a sky position that contains no (other) members of Tuc/Hor listed in either of these review articles. Therefore, we regard HIP 84642 as a possible rather than likely member of Tuc/Hor.

BD+44 3670. Guillout et al. (2009) and P. Guillout (2010, private communication) present data for this star including a lithium line $\mathrm{EW}=196 \mathrm{~m} \AA$. The photometric distance, radial velocity, and $U V W$ in Table 3 are from P. Guillout (2010, private communication).

HIP 116805. The star lies near the location of a typical Pleiades star on an A-star color-magnitude diagram.

\subsection{HR 8799}

HIP 114189 (=HR 8799) is known to be orbited by a multiple system of massive planets imaged by Marois et al. (2008, 2010) and by a massive dusty debris disk (Rhee et al. 2007b; Su et al. 2009). In Table 3, we place the star in the Columba Association based on its Galactic space motion and other age indicators mentioned below. If, as we suggest in Section 5.2, the nucleus of the Columba Association lies near $5^{\mathrm{h}} 30^{\mathrm{m}}$ R. A. and is $\sim 50$ pc from Earth, then currently HR 8799 is $\sim 70$ pc from the nucleus. A peculiar velocity of 2 $\mathrm{km}^{-1}$ over a period of $30 \mathrm{Myr}$ would produce this separation. Based on their Bayesian statistical analysis, Doyon et al. (2010) independently deduce that HR 8799 is a member of the Columba Association.
Recently, Currie et al. (2011) also deduce a young age for HR 8799; models of dynamical stability and planet evolution (cooling) lead them a preferred age near $30 \mathrm{Myr}$. The nearIR colors of the planets of HR 8799 differ from those of conventional (old) L- and T-type substellar objects, but are similar to that of the $\sim 6$ Jupiter mass 2 M1207b (Marois et al. 2008; Patience et al. 2010; Currie et al. 2011). As noted by Marois et al. (2010), evolutionary models for cooling $30 \mathrm{Myr}$ old planets suggest masses about six times that of Jupiter for the four known planets of HR 8799. Given the well-established age of 2M1207b ( 10 Myr), its unusual near-IR spectrum can most readily be attributed to atmospheric properties engendered by its low mass.

HR 8799 lies below the position of a typical Pleiades star on an A-star color-magnitude diagram; see HR 8799 plotted in Figure 5 of Zuckerman (2001). Because HR 8799 is a $\lambda$ Boo-type star with a peculiar surface composition, it probably should be plotted slightly to the right of where it appears in Zuckerman's Figure 5, but still below the Pleiades line in the figure. For example, with the $T_{\text {eff }}=7430 \mathrm{~K}$ derived by Gray $\&$ Kaye (1999), the star would plot near $B-V=0.31$. Relative to the locus of A-type Pleiades stars, HR 8799 plots as low or lower than all members of a sample of $\sim 20 \lambda$ Boo stars with Hipparcos measured parallaxes identified by R. Gray (2010, private communication). Again this is consistent with a young age.

HR 8799 has one of the most massive dusty debris disks known for any main-sequence star (Rhee et al. 2007b). The range of dust temperatures is extensive ( $\mathrm{Su}$ et al. 2009); in their preferred model, an inner warm dust belt with temperature $\sim 150 \mathrm{~K}$ extends between 6 and $15 \mathrm{AU}$ from the star. Marois et al. (2010) note that the inner edge of such a dust belt could be in a 4:1 mean motion resonance with planet HR 8799e while the outer edge must be closer to the star than 15 AU to avoid planet e's chaotic region. The location of the inner edge is determined only by a fit to the shorter wavelength portion of the Spitzer IRS spectrum combined with the assumption that the grains radiate like blackbodies at the relevant wavelengths (see Figures 3 and 4 in Su et al. 2009). Because the radius of a typical grain must be at least a few microns to avoid radiative blowout, this assumption is valid. Since the longer wavelength IRS emission is due to a blend of emission from the warm dust belt and cooler dust in an outer belt, the outer radius of the inner belt is not so well determined. It remains to be seen whether yet a fifth planet can be squeezed in between the warm dust belt and planet e (Hinkley et al. 2011).

$$
\text { 5.4. Argus }
$$

Torres et al. (2008) proposed a new association comprising more than 60 stars of which somewhat more than half are members of the open cluster IC 2391 , located $\sim 140$ pc from Earth. Torres et al. dub the non-IC 2391 stars "field Argus members" and, of these, $\sim$ half are more than $100 \mathrm{pc}$ from Earth. Indeed, only six of their field Argus stars are within the 70 pc radius sphere surrounding Earth that constrains our proposed Table 4 additions to Argus. In addition, whereas none of the 29 Argus field members proposed by Torres et al. are of spectral type earlier than F0, five of the Argus stars in Table 4 are A type. We therefore expect that many additional A-type Argus members will be identified beyond $66 \mathrm{pc}$ from Earth. Previously, Eggen (1991) proposed an IC 2391 Supercluster containing many early-type stars, including Table 2 stars HIP 98495 and HIP 57632 ( $\beta$ Leo, only 11 pc from Earth). In 
the future, some A-type members of Eggen's Supercluster more distant than $66 \mathrm{pc}$ are likely to be joined to the Argus Association defined by Torres et al. (2008).

In addition to early-type Argus field stars more distant from Earth than $\sim 65$ pc, IC 2391 contains some A- and B-type stars (e.g., Siegler et al. 2007). Siegler et al. consider 34 members of IC 2391 ranging among spectral types B through M. Only 12 stars in the Siegler sample are included among the 35 IC 2391 stars considered by Torres et al. (2008) to be "high probability members" of the Argus Association. Neither of these two papers cites the other which is understandable given that their submissions may have been nearly simultaneous. However, the Siegler et al. (2007) paper is also not cited in a 2009 paper by the Torres group (da Silva et al. 2009). In any event, we consider the Argus Association and IC 2391 in Section 5.5.2 in conjunction with Table 11.

Torres et al. (2008) suggest an age of 40 Myr for the Argus Association. Its large negative $U$ component of space motion distinguishes Argus from other young nearby moving groups. While Argus members listed by Torres et al. (2008) are deep in the southern hemisphere, Table 2 stars HIP 57632 and HIP 99770 are in the northern hemisphere.

The A-type stars HIP 50191, 57632, and 99770 all lie near the typical Pleiades star on an A-star color-magnitude diagram, while HIP 79797 and 98595 lie substantially below Pleiades stars. The F-type star HIP 68994 also lies well below Pleiades stars; for it, $f_{x}=-5.1$.

\subsection{Spitzer Observations}

Unveiling the evolution of dusty debris disks as a function of stellar age has been a major focus of Spitzer studies of mainsequence stars. Rebull et al. (2008) and Gaspar et al. (2009) each present tables listing stellar associations and clusters of known age and the fraction of such stars with Spitzer detected IR excess emission. Neither listing contains stars in the $A B$ Dor Association although a few such stars appear in Spitzer papers by Plavchan et al. (2009), Carpenter et al. (2009), and Hillenbrand et al. (2008). The Rebull et al. paper does include an entry for Tuc/Hor based on a few stars from her paper and from Smith et al. (2006), but many more Tuc/Hor stars are included in tables in the present paper.

\subsubsection{Individual SEDs}

Figures 1-7 display the SED for all stars in Tables 8-10 with definite or probable IR excess emission. MIPS fluxes presented in this paper are color corrected. The tables give stellar and dust parameters derived from our SED-fitting routine. When only an upper limit to the $70 \mu \mathrm{m}$ flux density is plotted, then an indicated dust temperature is a lower limit and the indicated dust luminosity is an upper limit. We consider here a few of the more difficult and interesting SEDs.

CD-60 416 (Table 9). The $24 \mu \mathrm{m}$ MIPS flux density and the IRS spectrum both lie slightly above the estimated photospheric flux. However, the IRS is noisy and is not rising toward long wavelengths, so we regard the apparent excess emission at $24 \mu \mathrm{m}$ as questionable.

HIP 16563 (Table 8, Figure 1). This is $\sim 10^{\prime \prime}$ binary. The M0 secondary appears to have $24 \mu \mathrm{m}$ emission that is $40 \%$ in excess of the photosphere. If so, then HIP $16563 \mathrm{~B}$ is one of only a handful of M-type stars with measurable excess IR emission. The primary is about a magnitude brighter than the secondary and appears to have a $24 \mu \mathrm{m}$ excess of about $30 \%$. Lestrade et al. (2006) found no evidence for cold dust at these stars. Because the binary nature of the star introduces additional complexities into the analysis, we regard as tentative the excesses at both stars.

HIP 30034 (Table 9, Figure 5). This is AB Pic, a star that has a companion, imaged with adaptive optics (AO), with mass ( $\sim 13.5$ Jupiter masses) that straddles the planet/brown dwarf boundary (Chauvin et al. 2005). Although the $8 \mu \mathrm{m}$ flux density measured with IRAC on Spitzer is on or very near the photosphere, there is evidence for excess emission in both the $12 \mu \mathrm{m} I R A S$ and $24 \mu \mathrm{m}$ MIPS channels. The MIPS excess is $\sim 25 \%$. The color-corrected IRAS $12 \mu \mathrm{m}$ flux density is $70 \pm 11$ $\mathrm{mJy}$ while the photosphere is $\sim 40 \mathrm{mJy}$.

HIP 32435 (Table 9, Figure 5). The shape of the IRS spectrum seems difficult to reconcile with the elevated $60 \mu \mathrm{m} I R A S$ point.

HIP 68994 (Table 10, Figure 7). This star lies precisely in the Galactic plane so that contamination by background IR sources is always a possibility. That said, we note the elevated AKARI $9 \mu \mathrm{m}$ flux density that, along with the $24 \mu \mathrm{m}$ MIPS measurement, suggests the presence of substantial quantities of warm dust particles.

HIP 114189 (Note to Table 9). The SED has been analyzed in great detail by Su et al. (2009), thus we do not present it here.

HIP 115738 (Table 8, Figure 2). The IRS spectrum of this A0 star indicates a rising SED from 10 to $30 \mu \mathrm{m}$, but with the MIPS $70 \mu \mathrm{m}$ flux density essentially back on the photosphere. We have not tried to fit a dust temperature.

\subsubsection{Evolution of Dusty Debris Disks with Time}

As mentioned in Section 4, because the Columba Association is about the same age as Tuc/Hor (30 Myr) we combine Spitzer observed stars from these associations into a single entry in Table 11. Both Rebull et al. (2008) and Gaspar et al. (2009) tabulate the percentage of stars with excess emission found by Spitzer in various nearby clusters and associations. For the five associations/clusters for which we agree with the fraction of members with excess 24 and $70 \mu \mathrm{m}$ emission given in Table 5 in Rebull et al. (2008), we cite their paper and adopt their quoted values in the right-hand column of Table 11; and similarly for the three clusters where we cite Gaspar et al. For the $24 \mu \mathrm{m}$ excess fraction in IC 2391, we follow both Gaspar et al. and Rebull et al. (who agree). For the Pleiades and for NGC 2451, we take excess fractions from the original (cited) Spitzer papers.

At $24 \mu \mathrm{m}$ wavelength Spitzer was sufficiently sensitive to detect the stellar photospheres. The entries in the third and fourth columns of Table 11 indicate that the fraction of stars that possess excess $24 \mu \mathrm{m}$ emission above the photosphere is rather constant at about $1 / 3$ between 8 and $~ 50$ Myr. Subsequently, this fraction declines to $\sim 15 \%$ at $\sim 100 \mathrm{Myr}$ and then to only a percent or two at $\sim 700 \mathrm{Myr}$.

At $70 \mu \mathrm{m}$, because the photospheric flux level is usually not reached in Spitzer observations, only lower limits to the percentage of stars with excess emission can be derived. This lower limit is about $1 / 3$ for ages between 8 and $50 \mathrm{Myr}$, i.e., comparable to the percentage of stars with excess $24 \mu \mathrm{m}$ at similar ages. For cluster stars of age $100 \mathrm{Myr}$ and greater the fraction that possess $70 \mu \mathrm{m}$ excess emission is much smaller than $1 / 3$.

\section{CONCLUSIONS}

We propose 35 star systems within $\sim 70$ pc of Earth as new members of previously identified young, moving groups. With but one exception, these 35 stars are brighter than 10th magnitude at the $V$ band. Thus, they should generally be excellent 
targets for extreme-AO and space-based, near-infrared, imaging searches for warm planets.

Among the 35 star systems are some that appear to have the same Galactic space motions and ages as stars in the previously proposed Columba and Argus field associations (Torres et al. 2008), but over a much wider range of right ascension and/or declination. It remains to be seen whether all such stars belong to these already defined kinematic groups or if additional young moving groups will need to be defined. Our unpublished optical spectroscopic observations of X-ray bright stars from Siding Spring and Lick observatories indicate that many young stars near Earth have space motions that differ from those of all moving groups and associations listed in Zuckerman \& Song (2004) and Torres et al. (2008).

We comment on many of the 35 star systems and also on some other stars that clearly are young but that do not seem to quite fit into known moving groups. One of the more interesting of the 35 stars is HR 8799 that is orbited by at least four giant planets at wide separations. The Galactic space motion of HR 8799 is in excellent agreement with that of the $30 \mathrm{Myr}$ old Columba Association and we present it as one of six northern hemisphere stars we are proposing as members of this association.

We present the first comprehensive consideration of Spitzer data for stars in the AB Doradus and Tucana/Horologium Associations. We also consider Spitzer results for nearby stars in the Columba and field Argus Associations. As young stars are wont to do, many of these stars display excess IR emission at 24 and/or $70 \mu \mathrm{m}$ wavelength. For a few stars, $24 \mu \mathrm{m}$ emission appears to dominate, thus suggesting the presence of warm dust particles. One such warm dust, solar-like star may be AB Pic which is known to be orbited by a distant companion with a mass that straddles the planet/brown dwarf mass boundary.

Combination of our Spitzer results with those for 11 other nearby clusters and associations illustrates the decay of dusty debris with time over the age range 8-750 Myr. For cluster/ association stars of ages 8 to $\sim 50 \mathrm{Myr}$ about $1 / 3$ display excess 24 and $70 \mu \mathrm{m}$ emission above the photosphere. The percentage with excess $24 \mu \mathrm{m}$ emission drops to $\sim 15 \%$ at $100 \mathrm{Myr}$ and then to only a few percent at $\sim 700$ Myr. Similarly, the percentage of stars with Spitzer detected excess emission at $70 \mu \mathrm{m}$ and age $>100$ Myr is much smaller than $1 / 3$.

We thank the referee for useful suggestions. This research was funded in part by NASA grants to UCLA and the University of Georgia.

\section{REFERENCES}

Aumann, H., et al. 1984, ApJ, 278, L23

Balog, Z., et al. 2009, ApJ, 698, 1989

Carpenter, J., et al. 2009, ApJS, 181, 197

Chauvin, G., et al. 2005, A\&A, 438, L29

Chauvin, G., et al. 2010, A\&A, 509, A52

Currie, T., et al. 2011, ApJ, 729, 128

Cutispoto, G., Pastori, L., Pasquini, L., de Medeiros, J., Tagliaferri, G., \& Andersen, J. 2002, A\&A, 384, 491

da Silva, L., Torres, C., de La Reza, R., Quast, G., Melo, C., \& Sterzik, M. 2009, A\&A, 508, 833

Doyon, R., Malo, L., Lafrenière, D., \& Artigau, E. 2010, in Proc. of Conf. In the Spirit of Lyot: The Direct Detection of Planets and Circumstellar Disks, ed. A. Boccaletti (Paris: LESIA/CNRS)

Eggen, O. J. 1991, AJ, 102, 2028

Engelbracht, C., et al. 2007, PASP, 119, 994

Fernandez, D., Figueras, F., \& Torra, J. 2008, A\&A, 480, 735

Gaspar, A., et al. 2009, ApJ, 697, 1578
Gizis, J. 2002, ApJ, 575, 484

Gontcharov, G. 2006, Astron. Lett., 32, 759

Gorlova, N., Rieke, G., Muzerolle, J., Stauffer, J., Siegler, N., Young, E., \& Stansberry, J. 2006, ApJ, 651, 1130

Gray, R., \& Kaye, A. 1999, AJ, 118, 2993

Guillout, P., et al. 2009, A\&A, 504, 829

Habing, H., et al. 2001, A\&A, 365, 545

Hauschildt, P., Allard, F., \& Baron, E. 1999, ApJ, 512, 377

Hillenbrand, L., et al. 2008, ApJ, 677, 630

Hinkley, S., Carpenter, J., Ireland, M., \& Kraus, A. 2011, ApJ, 730, L21

Kastner, J. H., Zuckerman, B., Weintraub, D., \& Forveille, T. 1997, Science, 277,67

Kiss, L., et al. 2011, MNRAS, 411, 117

Lestrade, J.-F., Wyatt, M., Bertoldi, F., Dent, W., \& Menten, K. 2006, A\&A, 460, 733

Looper, D., Bochanski, J., Burgasser, A., Mohanty, S., Mamajek, E., Faherty, J., West, A., \& Pitts, M. 2010a, AJ, 140, 1486

Looper, D., Burgasser, A., Kirkpatrick, J. D., \& Swift, B. 2007, ApJ, 669, L97

Looper, D., et al. 2010b, ApJ, 714, 45

Lopez-Santiago, J., Montes, D., Crespo-Chacon, I., \& Fernandez-Figueroa, M. 2006, ApJ, 643, 1160

Lopez-Santiago, J., et al. 2010, A\&A, 514, A97

Mamajek, E. 2005, ApJ, 634, 1385

Marois, C., Macintosh, B., Barman, T., Zuckerman, B., Song, I., Patience, J., Lafreniere, D., \& Doyon, R. 2008, Science, 322, 1348

Marois, C., Zuckerman, B., Konopacky, Q., Macintosh, B., \& Barman, T. 2010, Nature, 468, 1080

Melis, C., Zuckerman, B., Rhee, J., \& Song, I. 2010, ApJ, 717, L57

Moor, A., Abraham, P., Derekas, A., Kiss, Cs., Kiss, L., Apai, D., Grady, C., \& Henning, Th. 2006, ApJ, 644, 525

Moor, A., et al. 2009, ApJ, 700, L25

Patience, J., King, R., de Rosa, R., \& Marois, C. 2010, A\&A, 517, A76

Plavchan, P., Werner, M., Chen, C., Stapelfeldt, K., Su, K., Stauffer, J., \& Song, I. 2009, ApJ, 698, 1068

Rebull, L., et al. 2008, ApJ, 681, 1484

Rhee, J., Song, I., \& Zuckerman, B. 2007a, ApJ, 671, 616

Rhee, J., Song, I., Zuckerman, B., \& McElwain, M. 2007b, ApJ, 660, 1556

Rodriguez, D., Bessell, M., Zuckerman, B., \& Kastner, J. H. 2011, ApJ, 727, 62

Schlieder, J., Lepine, S., \& Simon, M. 2010, AJ, 140, 119

Scholz, R.-D., McCaughrean, M., Zinnecker, H., \& Lodieu, N. 2005, A\&A, 430, L49

Schroder, C., \& Schmitt, J. 2007, A\&A, 475, 677

Shkolnik, E., Liu, M., Reid, I. N., Dupuy, T., \& Weinberger, A. 2011, ApJ, 727,

Siegler, N., Muzerolle, J., Young, E., Rieke, G., Mamajek, E., Trilling, D., Gorlova, N., \& Su, K. 2007, ApJ, 654, 580

Silverstone, M. 2000, PhD thesis, Univ. California Los Angeles

Smith, P., Hines, D., Low, F., Gehrz, R., Polomski, E., \& Woodward, C. 2006, ApJ, 644, 125

Song, I., Zuckerman, B., \& Bessell, M. 2003, ApJ, 599, 342 (erratum 603, 804 [2004])

Su, K., et al. 2009, ApJ, 705, 314

Torres, C., da Silva, L., Quast, G., de la Reza, R., \& Jilinski, E. 2000, AJ, 120 , 1410

Torres, C., Quast, G., da Silva, L., de la Reza, R., Melo, C., \& Sterzik, M. 2006, A\&A, 460, 695

Torres, C., Quast, G., Melo, C., \& Sterzik, M. 2008, in Handbook of Star Forming Regions, Volume II: The Southern Sky, ed. B. Reipurth (ASP Monograph Publ., Vol. 5; San Francisco, CA: ASP), 757

Trilling, D., et al. 2008, ApJ, 674, 1086

Voges, W., et al. 1999, A\&A, 349, 389

Voges, W., et al. 2000, IAU Circ., 7432, 3

Waite, I., Carter, B., Marsden, S., \& Mengel, M. 2005, PASA, 22, 29

Webb, R., Zuckerman, B., Platais, I., Patience, J., White, R. J., Schwartz, M., \& McCarthy, C. 1999, ApJ, 512, L63

Wichmann, R., Schmitt, J. H. M. M., \& Hubrig, S. 2003, A\&A, 399, 983

Zuckerman, B. 2001, ARA\&A, 39, 549

Zuckerman, B., Bessell, M. S., Song, I., \& Kim, S. 2006, ApJ, 649, L115

Zuckerman, B., \& Song, I. 2004, ARA\&A, 42, 685

Zuckerman, B., Song, I., \& Bessell, M. 2004, ApJ, 613, L65

Zuckerman, B., Song, I., Bessell, M., \& Webb, R. 2001a, ApJ, 562, L87

Zuckerman, B., Song, I., \& Webb, R. 2001b, ApJ, 559, 388

Zuckerman, B., \& Webb, R. 2000, ApJ, 535, 959

Zuckerman, B., Webb, R., Schwartz, M., \& Becklin, E. E. 2001c, ApJ, 549, L233 